\title{
Myocardial Electrical Propagation in Patients with Idiopathic Dilated Cardiomyopathy
}

Kelley P. Anderson, Richard Walker, Paul Urie, Philip R. Ershler, Robert L. Lux, and Shreekant V. Karwandee

Cardiolog!' Division, and Department of Cardiothoracic Surger!; University of Utah Medical Center and Nora Eccles Harrison Cardiovascular Training and Research Institute, University of Utah, Salt Lake City, Utah 84132

\begin{abstract}
Myocardial propagation may contribute to fatal arrhythmias in patients with idiopathic dilated cardiomyopathy (IDC). We examined this property in 15 patients with IDC undergoing cardiac transplantation and in 14 control subjects. An $8 \times 8$ array with electrodes $2 \mathrm{~mm}$ apart was used to determine the electrical activation sequence over a small region of the left ventricular surface. Tissue from the area beneath the electrode array was examined in the patients with IDC. The patients with IDC could be divided into three groups. Group I $(n=7)$ had activation patterns and estimates of longitudinal $\left(\theta_{L}\right.$ $=0.84 \pm 0.09 \mathrm{~m} / \mathrm{s})$ and transverse $\left(\theta_{T}=0.23 \pm 0.05 \mathrm{~m} / \mathrm{s}\right)$ conduction velocities that were no different from controls $\left(\theta_{L}\right.$ $\left.=0.80 \pm 0.08 \mathrm{~m} / \mathrm{s}, \theta_{\mathrm{T}}=0.23 \pm 0.03 \mathrm{~m} / \mathrm{s}\right)$. Group II $(n=4) \mathrm{had}$ fractionated electrograms and disturbed transverse conduction with normal longitudinal activation, features characteristic of nonuniform anisotropic properties. Two of the control patients also had this pattern. Group III $(n=4)$ had fractionated potentials and severely disturbed transverse and longitudinal propagation. The amount of myocardial fibrosis correlated with the severity of abnormal propagation. We conclude that $(a)$ severe contractile dysfunction is not necessarily accompanied by changes in propagation, and $(b)$ nonuniform anisotropic propagation is present in a large proportion of patients with IDC and could underlie ventricular arrhythmias in this disorder. ( $J$. Clin. Invest. 1993. 92:122-140.) Key words: arrhythmia • cardiomyopathy • electrophysiology • myocardium • sudden death
\end{abstract}

\section{Introduction}

Sudden death occurs frequently in patients with idiopathic dilated cardiomyopathy (IDC), ${ }^{1}$ accounting for about half of the deaths in this disorder $(1,2)$. There is evidence that ventricular arrhythmias are a cause of sudden death in IDC (1). Unfortunately, a major clinical problem persists because treatment that

This report was presented in part in abstract form at the 62nd American Heart Association Scientific Sessions, New Orleans, LA 1989.

Address reprint requests to Dr. Kelley P. Anderson, Cardiology Division, University of Utah Medical Center, Salt Lake City, UT 84132.

Received for publication 11 June 1992 and in revised form 30 December 1992.

1. Abbreviations used in this paper: IDC, idiopathic dilated cardiomyopathy; NAI, nonuniform activation index; PVC, premature ventricular contraction; RMS, root mean square; ROC, receiver operating characteristic; TAT, total activation time; VT, ventricular tachycardia.

J. Clin. Invest.

(C) The American Society for Clinical Investigation, Inc.

$0021-9738 / 93 / 07 / 122 / 19 \$ 2.00$

Volume 92. July 1993, 122-140 could be directed toward large numbers of patients, such as antiarrhythmic drugs, has not been shown to be uniformly effective $(2,3)$, and highly specific predictors of sudden death, which could be used to target therapies such as cardiac transplantation or defibrillator implantation, have not been identified $(1,2)$. Therefore, there is a pressing need for better understanding of the factors that predispose patients with IDC to malignant ventricular tachyarrhythmias.

Much of what is known about ventricular arrhythmias is based on studies of patients with ischemic heart disease and animal models of myocardial infarction. In these studies reentry has been shown to be an important mechanism of arrhythmias, and abnormalities of conduction appear to play a principal role in their genesis (4). Much less is known about arrhythmia mechanisms in patients with IDC. There is some evidence of disturbed conduction in patients with IDC $(2,5-8)$, and there is considerable evidence of abnormal cellular function (9-11), abnormal passive electrical properties $(12,13)$, and abnormal morphologic features that could contribute to abnormal propagation (14-24). However, we are not aware of any previous attempts to systematically examine the spread of electrical activity in cardiomyopathic states in humans or in animal models. Because of the large number of abnormalities that could affect conduction in patients with IDC, a model of myocardial propagation was used to predict the effects that reported functional and structural changes would have on myocardial propagation. The hypothesis was that electrical propagation in the myocardium of patients with IDC would differ from that in control subjects, and that the changes would conform to patterns predicted by the model.

\section{Methods}

\section{Theory}

Our predictions about the behavior of propagating wavefronts in diseased myocardium were drawn from two sources, empirical data and models of propagation. Neither is completely satisfactory since neither can imitate diseased tissue perfectly and for several properties there is no suitable published information from either source. To supplement existing evidence, we used an analytic model of propagation derived from continuous cable theory by Keener (25):

$$
\begin{aligned}
& \theta=\frac{M_{\mathrm{f}} L}{C_{\mathrm{m}} R_{\mathrm{m}}} \sqrt{\frac{1}{r+q_{\mathrm{e}}+q_{\mathrm{i}}}}, \\
& \text { where } r=\frac{S r_{\mathrm{g}}}{R_{\mathrm{m}}} \cdot q_{\mathrm{i}}=\frac{S L^{2} R_{\mathrm{i}}}{V_{\mathrm{if}} R_{\mathrm{m}}} \cdot q_{\mathrm{e}}=\frac{S L^{2} R_{\mathrm{e}}}{V_{\mathrm{e}} R_{\mathrm{m}}},
\end{aligned}
$$

and where $\theta$ is the effective conduction velocity $(26), M_{\mathrm{f}}$ is a membrane function that incorporates excitability, ionic currents, and related phenomena (25), $L$ is the length of the myocyte in the direction of propagation, $C_{\mathrm{m}}$ is the membrane capacitance, $R_{\mathrm{m}}$ is the membrane resistance, $S$ is the membrane surface area of a single myocyte, $R_{\mathrm{g}}$ is the gap junctional resistance, $R_{\mathrm{i}}$ is the cytoplasmic resistance, $V_{\mathrm{i}}$ is the volume of a single cardiac cell, $R_{\mathrm{c}}$ is the resistance of the extracellular medium, and $V_{\mathrm{e}}$ is the volume of the extracellular medium. It is as- 
sumed that propagation can be represented by a plane wave traveling either parallel to fiber orientation or perpendicular to it, that there are only two types of currents, transmembrane and axial, and that voltage potentials are uniformly distributed along the cell and in the extracellular space perpendicular to the direction of propagation $(25,27,28)$. Moreover, it is assumed that the parameters given in Eq. 1 are independent of each other. Violations of the assumptions of continuous cable theory are well known $(25,27)$; in fact, Eq. 1 was derived primarily to demonstrate the inadequacies of this model in comparison with a model based on the discrete nature of myocardial propagation (25). However, this model suited our purpose, which was to complement previously reported results to develop working hypotheses about the patterns of propagation that might be expected for the variety of structural and electrical alterations anticipated in myopathic cardiac tissue rather than to derive specific results. The advantage of this formulation is that it presents propagation velocity as a function of the important cable parameters which permits an intuitive appreciation of the relation between electrical properties without the requirement of complex numerical simulations.

The baseline values of the parameters of the model were obtained from the literature or from reasonable estimates, and all parameter values were varied to assess the effect on conduction velocities: The length of the cell for propagation longitudinal to fiber orientation: $L_{\mathrm{L}}$ $=120 \mu \mathrm{m}(0.012 \mathrm{~cm})$, the length of the cell (diameter of the cell) for propagation transverse to fiber orientation: $L_{\mathrm{T}}=20 \mu \mathrm{m}(0.002 \mathrm{~cm})$, which are within the normal range for mammalian ventricular myocytes (29). The volume of a single myocyte was calculated from the length and diameter assuming a cylindrical shape: $V_{\mathrm{i}}=3.77 \times 10^{-8}$ $\mathrm{cm}^{3}$. The membrane surface area of a myocyte was obtained by multiplying the surface area of the cylinder by 10 to estimate the additional surface provided by the transverse tubular system $(27,30): S=7.54$ $\times 10^{-4} \mathrm{~cm}^{2}$. The volume of the extracellular space was assumed to be approximately one-sixth of the volume of the myocyte to represent relatively tight packing of cells in normal myocardium: $V_{\mathrm{e}}=6.0 \times 10^{-9}$ $\mathrm{cm}^{3}(31)$. Specific membrane capacitance: $C_{\mathrm{m}}=1 \mu \mathrm{F} / \mathrm{cm}^{2}(30,32)$. Specific membrane resistance: $R_{\mathrm{m}}=9,000 \Omega \mathrm{cm}^{2}(30)$. Intracellular resistance: $R_{\mathrm{i}}=400 \Omega \mathrm{cm}(30,33,34)$. Extracellular resistance: $R_{\mathrm{e}}=50$ $\Omega \mathrm{cm}(30)$. The gap junctional resistance in the longitudinal direction $r_{\mathrm{BL}}=0.5 \mathrm{~m} \Omega$; in the transverse direction $r_{\mathrm{gT}}=1 \mathrm{M} \Omega(33,34)$. With the membrane function parameter arbitrarily set $M_{\mathrm{f}}=30$, these values resulted in effective longitudinal conduction velocity $\theta_{\mathrm{L}}=0.77 \mathrm{~m} / \mathrm{s}$, effective transverse conduction velocity $\theta_{\mathrm{T}}=0.23 \mathrm{~m} / \mathrm{s}$, and $\theta_{\mathrm{L}} / \theta_{\mathrm{T}}$ $=3.5$, which corresponded well to the average findings in control subjects.

Six general patterns of myocardial activation, which might be observed based on known alterations in myopathic states, were derived from the model and previous experimental results:

Pattern $A$. This first pattern is of uniform anisotropic propagation (35) with values of $\theta_{\mathbf{L}}, \theta_{\mathrm{T}}$, and $\theta_{\mathrm{L}} / \theta_{\mathrm{T}}$ not different from controls. Although few studies have been performed in human ventricular myocardium, studies in other species demonstrate that pattern A is typical of activation at the left ventricular surface $(36,37)$.

Pattern $B$. The second pattern is of uniform anisotropic propagation with proportionately equal reductions of $\theta_{\mathrm{L}}$ and $\theta_{\mathrm{T}}$ and unchanged $\theta_{\mathrm{L}} / \theta_{\mathrm{T}}$ ( pattern $\mathrm{B} 1$ ), or proportionately greater reduction of $\theta_{\mathrm{L}}$ than $\theta_{\mathrm{T}}$ and decreased $\theta_{\mathrm{L}} / \theta_{\mathrm{T}}$ (pattern B2). Reductions in resting membrane potential or in action potential upstroke velocity $\left(\dot{\mathrm{V}}_{\max }\right)$ correspond to lower values of $M_{\mathrm{f}}$ and should result in lower $\theta_{\mathrm{L}}$ and $\theta_{\mathrm{T}}$ with no change in $\theta_{\mathrm{L}} / \theta_{\mathrm{T}}$ (pattern $\mathrm{B} 1$ ) according to the model. Experimental interventions that alter these properties, however, have sometimes resulted in pattern B2 $(37-43)$ as well as B1 $(28,44)$. The model predicts that increases in $\mathrm{C}_{\mathrm{m}}, \mathrm{R}_{\mathrm{m}}$, or $S$ produce the B1 pattern (Fig. $1 A$ ). The B2 pattern is expected with increases of cytoplasmic $\left(R_{\mathrm{i}}\right)$ and extracellular $\left(R_{e}\right)$ resistance (Fig. $1 B$ ). We could find no reports of experimental manipulations that supported or refuted the predictions of the model for two-dimensional spread of activation with respect to changes in $C_{\mathrm{m}}$, $R_{\mathrm{m}}, S, R_{\mathrm{i}}$, or $R_{\mathrm{e}}$.
Pattern $C$. Third is a pattern of uniform anisotropic propagation with increases of $\theta_{\mathrm{L}}$ and $\theta_{\mathrm{T}}$. A number of changes known to occur in the myocardium of patients with IDC would be expected actually to increase $\theta$. The model predicts increases in $\theta_{\mathrm{L}}$ and $\theta_{\mathrm{T}}$ with no change in $\theta_{\mathrm{L}} / \theta_{\mathrm{T}}$ (pattern $\mathrm{C} 1$ ) with reduced $S$ due to shrinkage of the transversetubular system (Fig. $1 \mathrm{~A}$ ). It has been suggested that reduced numbers of myofibrils $(10,19,22,24,45)$ decreases $R_{i}(30,46)$. Reductions of $R_{\mathrm{i}}$ or $R_{\mathrm{c}}$ increase $\theta_{\mathrm{L}}, \theta_{\mathrm{T}}$, and $\theta_{\mathrm{L}} / \theta_{\mathrm{T}}$ (pattern C2, Fig. $1 B$ ). An increase of $V_{\mathrm{e}}$ resulting from expansion of the interstitial space increases $\theta_{\mathrm{L}}$ and $\theta_{\mathrm{L}} / \theta_{\mathrm{T}}$, with only a slight increase of $\theta_{\mathrm{T}}$ (Fig. $1 C$ ). The pattern that accompanies myocyte hypertrophy depends whether the change in myocyte volume $\left(V_{\mathrm{i}}\right)$ is due to lengthening or widening of the cell. Elevation of $V_{\mathrm{i}}$ due to increased cell length $\left(L_{\mathrm{L}}\right)$ increases $\theta_{\mathrm{L}}$ and $\theta_{\mathrm{L}} / \theta_{\mathrm{T}}$, while $\theta_{\mathrm{T}}$ changes little (pattern C2). In contrast, $\theta_{\mathrm{L}} / \theta_{\mathrm{T}}$ declines (pattern $\mathrm{C} 3$ ) with larger cell diameter (Fig. $1 D$ ). The model predicts that proportional increases in $L_{\mathrm{L}}$ and $L_{\mathrm{T}}$ produce a $\mathrm{C} 3$ pattern with a gradual fall of $\theta_{\mathrm{L}} / \theta_{\mathrm{T}}$ as $\theta_{\mathrm{L}}$ and $\theta_{\mathrm{T}}$ rise (Fig. $1 E$ ).

Pattern $D$. The fourth pattern is one of uniform anisotropic propagation with decreased $\theta_{\mathrm{T}}$, increased $\theta_{\mathrm{L}} / \theta_{\mathrm{T}}$, and slight decreases or no change of $\theta_{\mathbf{L}}$. This is the pattern predicted with a generalized increase in gap junctional resistance with proportionately equal changes in the longitudinal $\left(r_{\mathrm{gL}}\right)$ and transverse $\left(r_{\mathrm{gT}}\right)$ directions (Fig. $\left.1 F\right)$, and has been observed experimentally $(28,47,48)$.

Pattern E. Next is a nonuniform anisotropic pattern: variable reductions in $\theta_{\mathrm{T}}$, normal $\theta_{\mathrm{L}}$ and fractionated electrograms during transverse propagation. This pattern of propagation was identified by Spach and co-workers who proposed that such behavior results from reduced "side-to-side" electrical connections $(35,49)$.

Pattern F. Last is a nonuniform anisotropic pattern with variable reductions of $\theta_{\mathrm{L}}$ and $\theta_{\mathrm{T}}$ and fractionated electrograms. This pattern differs from pattern $E$ in that both longitudinal and transverse propagation are disturbed in an irregular pattern. It has been observed in the border zone of experimental healed myocardial infarction $(50,51)$.

\section{Patients}

The study subjects consisted of patients with IDC undergoing cardiac transplantation for severe congestive heart failure. Coronary artery disease, valvular heart disease, hypertension, diabetes mellitus, alcohol abuse, hypertrophic cardiomyopathy, and infiltrative cardiomyopaties were excluded in all patients by an extensive evaluation which included a history, physical examination, electrocardiogram and, when indicated, echocardiography, radionuclide angiography, coronary and left ventricular angiography, and myocardial biopsy. The control subjects consisted of patients with normal cardiac function undergoing operative correction of supraventricular arrhythmias. None of the patients were receiving antiarrhythmic drugs or had ever received amiodarone. Informed written consent to the protocol approved by the University of Utah Institutional Review Board was obtained for all patients.

\section{Electrophysiologic data}

Electrical activation data were obtained from an electrode array consisting of 64 silver electrodes $(0.6 \mathrm{~mm}$ in diameter, $2-\mathrm{mm}$ interelectrode distance) in an $8 \times 8$ square pattern embedded in a rigid epoxy plaque. The recordings were obtained in unipolar mode referenced to a needle in the right chest wall. The data acquisition and analysis system has been described previously (52). Electrograms were recorded at a band width of $0.03-500 \mathrm{~Hz}$, sampled at a $1 \mathrm{kHz}$ rate, and digitized by a 12-bit analog-to-digital converter. The intraoperative collection of data was standardized as far as possible to produce uniform experimental conditions but for ethical and practical reasons was limited to $20 \mathrm{~min}$. Electrophysiological recordings were obtained before the initiation of cardiopulmonary bypass. The closely spaced electrode plaque array was placed on the left ventricular epicardial surface adjacent to the left anterior descending artery two-thirds of the way from the base of the heart toward the apex and thus several centimeters away from any accessory atrioventricular connections. Unipolar cathodal or bipolar stimulation was performed from each edge and from the middle of the 

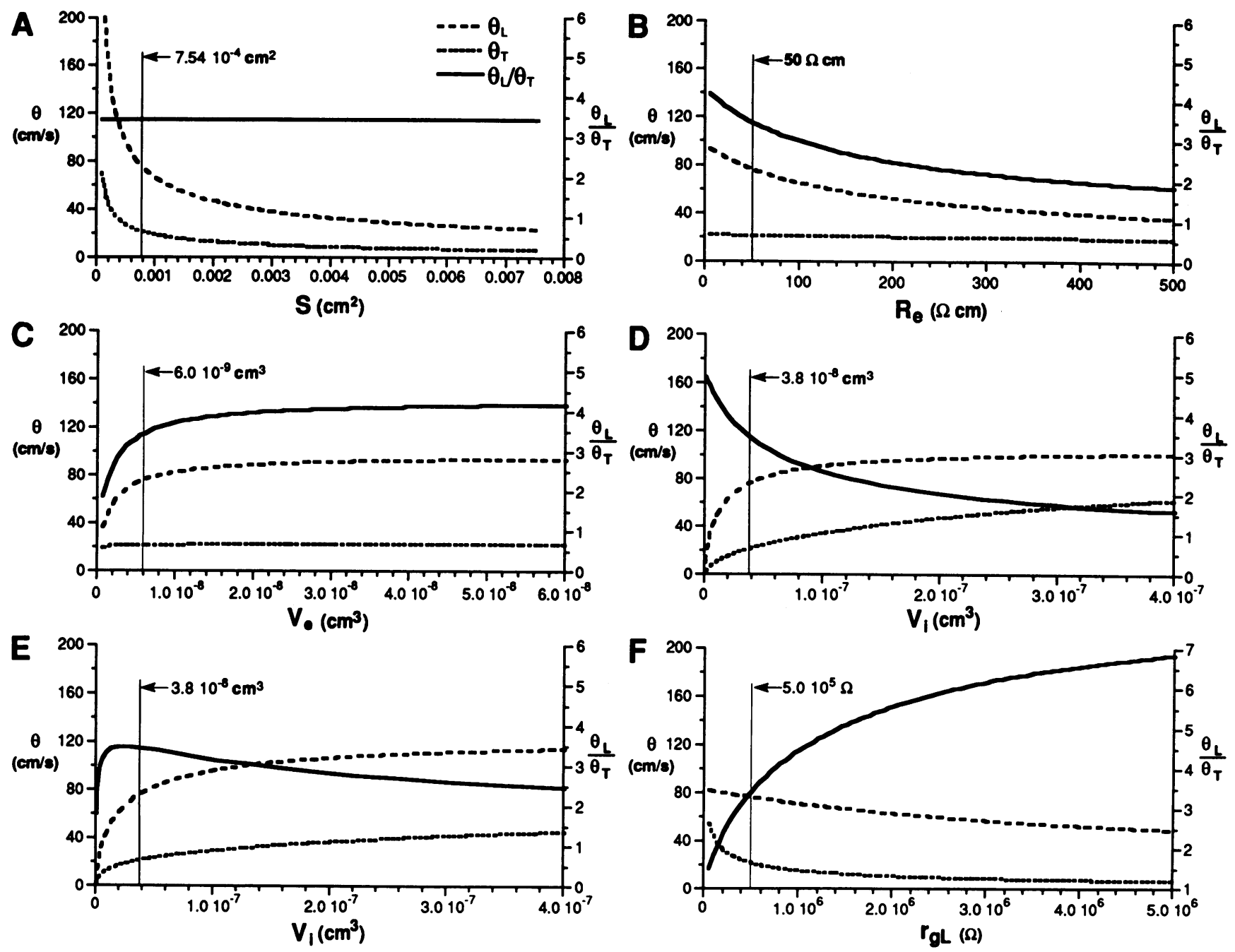

Figure 1. Plots of longitudinal conduction velocity $\left(\theta_{\mathrm{L}}\right)$, transverse conduction velocity $\left(\theta_{\mathrm{T}}\right)$, and the ratio of the velocities $\left(\theta_{\mathrm{L}} / \theta_{\mathrm{T}}\right)$ as functions of several electrophysiologic variables according to a continuous cable model (Eq. 1). The "normal" value of each variable is indicated by a vertical line. $\theta_{\mathrm{L}}, \theta_{\mathrm{T}}$, and $\theta_{\mathrm{L}} / \theta_{\mathrm{T}}$ are plotted as functions of the given variable from 0.1 to 10 times the normal value in panels $A, B$, and $C$. ( $\left.A\right)$ Increasing membrane surface area $(S)$ results in proportionately equal reductions of $\theta_{\mathrm{L}}$ and $\theta_{\mathrm{T}} ; \theta_{\mathrm{L}} / \theta_{\mathrm{T}}$ remains unchanged (pattern B1). Decreasing $S$ increases $\theta_{\mathrm{L}}$ and $\theta_{\mathrm{T}}$ with no change of $\theta_{\mathrm{L}} / \theta_{\mathrm{T}}$ (pattern $\mathrm{C} 1$ ). (B) Increasing $R_{\mathrm{e}}$ causes a greater reduction of $\theta_{\mathrm{L}}$ than $\theta_{\mathrm{T}}$ so $\theta_{\mathrm{L}} / \theta_{\mathrm{T}}$ declines $($ pattern B2). Decreasing $R_{\mathrm{e}}$ increases $\theta_{\mathrm{L}}$ and $\theta_{\mathrm{L}} / \theta_{\mathrm{T}}$ while $\theta_{\mathrm{T}}$ increases slightly (pattern $\mathrm{C} 2$ ). (C) Increasing $V_{\mathrm{e}}$ results in a rise of $\theta_{\mathrm{L}}$ and $\theta_{\mathrm{L}} / \theta_{\mathrm{T}}$ while $\theta_{\mathrm{T}}$ increases only slightly (pattern C3). (D) Myocyte volume $\left(V_{\mathrm{i}}\right)$, which is a function of the length $\left(L_{\mathrm{L}}\right)$ and diameter $\left(L_{\mathrm{T}}\right)$ of the cell and is assumed to be cylindrical $\left(V_{\mathrm{i}}=\pi \mathrm{L}_{\mathrm{L}} \mathrm{L}_{\mathrm{T}}{ }^{2}\right)$ was varied by changing only $L_{\mathrm{T}}$, keeping $L_{\mathrm{L}}$ constant. As $V_{\mathrm{i}}$ increases, $\theta_{\mathrm{T}}$ and $\theta_{\mathrm{L}} / \theta_{\mathrm{T}}$ increase, while $\theta_{\mathrm{L}}$ changes only slightly. $(E)$ This plot was obtained by varying both $L_{\mathrm{L}}$ and $L_{\mathrm{T}}$ but maintaining a constant ratio of $L_{\mathrm{L}}$ and $L_{\mathrm{T}} ; V_{\mathrm{i}}$ remains a function of $L_{\mathrm{L}}$ and $L_{\mathrm{T}}$. As $V_{\mathrm{i}}$ increases both $\theta_{\mathrm{L}}$ and $\theta_{\mathrm{T}}$ rise, while $\theta_{\mathrm{L}} / \theta_{\mathrm{T}}$ declines slightly. $(F) \theta_{\mathrm{L}}, \theta_{\mathrm{T}}$ and $\theta_{\mathrm{L}} / \theta_{\mathrm{T}}$ as a function of longitudinal gap junctional resistance $\left(r_{\mathrm{gL}}\right)$. This plot was obtained varying $r_{\mathrm{gL}}$ between 0.1 and 10 times the normal value $\left(5 \times 10^{5}\right)$, and keeping the ratio of longitudinal and transverse gap junctional resistance constant $\left(r_{\mathrm{gL}} / r_{\mathrm{gT}}=0.5\right)$.

array using rectangular impulses $2 \mathrm{~ms}$ in duration at twice diastolic threshold current. Data were acquired after pacing for $10 \mathrm{~s}$ at 150 beats/min. In some cases recordings were obtained after premature stimuli delivered after eight driven beats at 120 or 150 beats $/ \mathrm{min}$. In the patients with IDC the location of the plaque was marked before removal with a gentian violet pen.

\section{Histologic sections}

After the heart was removed in patients with IDC, the full thickness of myocardium beneath the plaque electrode array was excised and fixed in $10 \%$ formalin, embedded in paraffin, and stained with hematoxylin and eosin and Masson's trichrome stains. The subepicardial tissue was examined to determine the orientation and alignment of myocardial fibers and the pattern of fibrosis. The proportion of fibrous tissue in a given field was determined using a CUE 2 Image Analysis System
(Olympus Corp., Cherry Hill, NJ). The color and gray scale characteristics of the fibrous tissue stained with trichrome were determined and automatically highlighted by the analysis system. After the computerselected regions were verified and corrected by the operator, the fraction of fibrous tissue was computed by the analysis system.

\section{Determination of activation}

The minimum value (i.e., the greatest negative value) of the first temporal derivative of the extracellular potential $\left(\dot{\phi}_{\min }\right)$ has been shown to correspond with the most rapid phase of the action potential upstroke (49). Hence, the time of the deflection $\left(T_{\mathrm{D}}\right)$ was defined as the time at which $\dot{\phi}_{\min }$ occurred. Numerous possible tests for activation were compared to identify the test with the greatest ability to discriminate local from nonlocal electrical activity using methods previously described in detail (53). 


\section{Conduction velocity}

Strict measurement of conduction velocity requires knowledge of the entire path of activation in three dimensions. However, consistent values of the "effective" conduction velocity $(\theta)$ can be obtained from recordings obtained in two dimensions if care is taken to include only propagation in the recording plane $(37,42,54)$. This is primarily a problem for propagation transverse to fiber orientation. Human left ventricular myocardium tends to be arranged in "onion skin" layers where the fiber orientation of each successive layer is slightly rotated (55). Thus, myocardial tissue subject to activation via transverse propagation at the surface can be "preexcited" by a wavefront that travels more rapidly in a subepicardial layer which can lead to an overestimation of $\theta(56)$. This can be avoided by excluding areas distant from the stimulation site which demonstrate widening of isochrone contours. Two methods of estimating $\theta$ were used: In method A, longitudinal and transverse $\theta\left(\theta_{\mathrm{AL}}\right.$ and $\theta_{\mathrm{AT}}$, respectively $)$ were calculated by dividing the distance between two electrode sites by the difference in activation times at those sites $(35,37,42)$. The sites used in the calculation were those that determined a line normal to the wavefront in the longitudinal (fast) and transverse (slow) directions (Fig. 2). In some cases, however, identification of a line perpendicular to the wavefront required some judgement and in some cases there were no sites that were exactly collinear with the line. For these reasons a second method of estimating $\theta$ was obtained from the velocity of a plane wave across four adjacent sites $(57,58)$ :

$\theta_{\mathrm{B}}=\sqrt{\frac{D^{2}}{(a-b)^{2}+(c-d)^{2}}}$,

where $D$ is twice distance between electrodes $(4 \mathrm{~mm})$ and $a, b, c$, and $d$ are the activation times at sites superior, inferior, to the left, and to the right of the given site, respectively. Method $B$ estimates of longitudinal and transverse conduction velocities $\left(\theta_{\mathrm{BL}}\right.$ and $\theta_{\mathrm{BT}}$, respectively) consisted of the mean of values computed at several sites along the longitudinal and transverse directions (Fig. 2). For both methods, sites within $4 \mathrm{~mm}$ of the stimulation site were not used to avoid the virtual cathode effect (59). Areas of nonuniform activation (sudden narrowing or widening of isochrones) were also avoided.

\section{Nonuniform activation index (NAI)}

Nonuniformity of activation was quantified by using the NAI, which has been described in detail previously (56). In brief, the NAI at an electrode site $x\left(\mathrm{NAI}_{x}\right)$ is the sum of the second spatial derivatives of activation times in four directions times the electrode spacing. The second central difference formula was used to estimate the derivative terms with $\Delta \mathrm{x}$ as the electrode spacing:

$$
\begin{aligned}
& N A I_{X}=\frac{A-X}{\sqrt{2} \Delta x}+\frac{B-X}{\Delta x}+\frac{C-X}{\sqrt{2} \Delta x}+\frac{D-X}{\Delta x}+\frac{E-X}{\sqrt{2} \Delta x} \\
& +\frac{F-X}{\Delta x}+\frac{G-X}{\sqrt{2} \Delta x}+\frac{H-X}{\Delta x},
\end{aligned}
$$

where $X$ is the activation time at site $x$, and $A, B, C, D, E, F, G$, and $H$ are the activations times of the sites adjacent to site $x$. A positive $\mathrm{NAI}_{x}$ occurs with deceleration of activation, i.e., when isochrones get narrower. A negative value indicates acceleration of activation, or widening of isochrones. In this study NAI was the average of the absolute values the $\mathrm{NAI}_{x}$ 's computed at every possible electrode site during pacing from each edge of the electrode plaque.

\section{Statistical analysis}

Receiver operating characteristic (ROC) curves were used to compare the candidate tests for activation using methods described previously $(53,60)$. The quality of sensitivity $\left(Q_{\mathrm{SE}}\right)$, specificity $\left(Q_{\mathrm{SP}}\right)$, and efficiency $\left(Q_{\mathrm{E}}\right)$ were calculated as proposed by $\operatorname{Kraemer}(60)$. $Q_{\mathrm{SE}}$ was plotted against $Q_{\mathrm{SP}}$ to obtain the "quality" ROC. Student's $t$ test was used for comparison of continuous variables. The Kolmogorov-Smirnov test was used when the distributions were not normal. Differences were considered significant when $P \leq 0.05$.

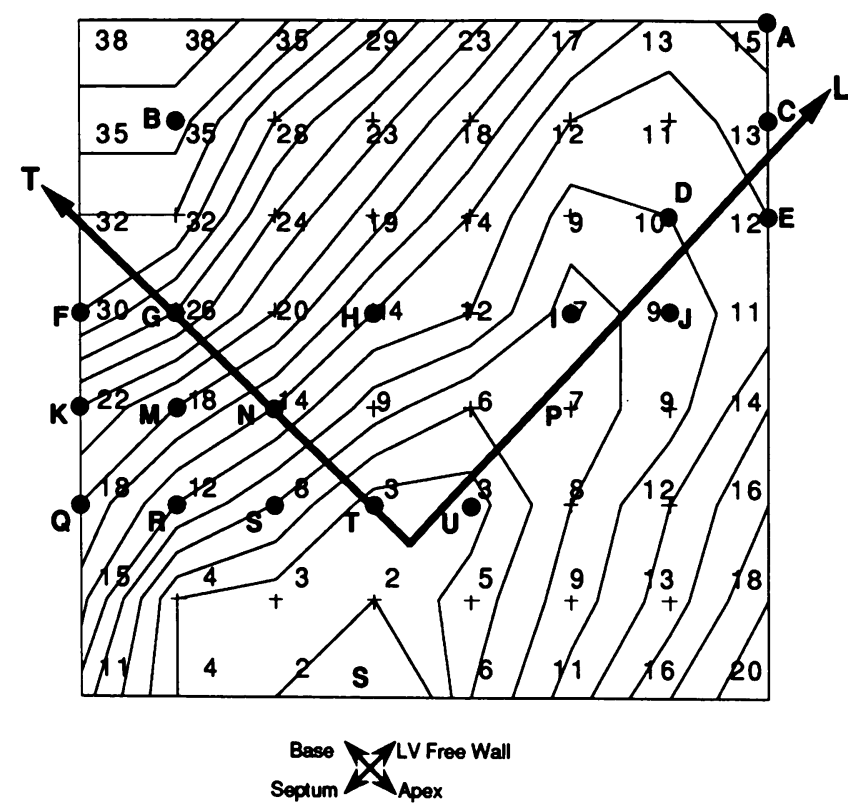

Figure 2. Electrical activation map based on recordings from the 64 electrode plaque array placed on the free wall of the left ventricular surface near the anterior interventricular groove in a patient with IDC. Its orientation with respect to the septum, left free wall, apex, and base of the heart is indicated in the diagram below the map. The isochrone contours are drawn by the method of linear interpolation by a computer program based on the activation times. The interval between isochrones is $2 \mathrm{~ms}$. The peripheral electrodes sites are on the border of the map diagram, the inner electrode sites are depicted by + , and the labeled electrode sites $(A, B, C$, etc.) are indicated by filled circles $(\bullet)$. The numbers adjacent to each electrode site are the activation times relative to the time of the stimulus artifact. The program does not attempt to smooth or to draw curved contours; sites with simultaneous activation are connected with straight lines resulting in somewhat jagged contours. The printed numbers are absent at sites where the recording channel malfunctioned or where the stimulus artifact obscured the electrograms. Stimulation was performed at a cycle length of $400 \mathrm{~ms}$ from an electrode along the lower edge (S). The sudden widening of isochrone contours in the upper left corner of the map probably resulted from rapid subepicardial spread of activation that activated several sites in that region nearly simultaneously. The lines with arrowheads indicate the directions of longitudinal $(L)$ and transverse $(T)$ propagation ( confirmed by histological examination ), and were used to select the electrode sites for estimating conduction velocity. Effective longitudinal conduction velocity obtained by method $\mathrm{A}\left(\theta_{\mathrm{AL}}\right)$ was calculated between sites $\mathrm{U}$ and $\mathrm{C}, \theta_{\mathrm{AL}}=1.00$ $\mathrm{m} / \mathrm{s}$. Note that this value of $\theta_{\mathrm{AL}}$ is greater than would have been obtained if sites $U$ and $A(\theta=0.83 \mathrm{~m} / \mathrm{s})$, or sites $U$ and $E(\theta=0.94$ $\mathrm{m} / \mathrm{s}$ ) had been used. Logically, $\theta_{\mathrm{AL}}$ should be best estimated by the most rapid value normal to the activation front because lower values may reflect some component of transverse conduction. Longitudinal conduction velocity estimated by method $\mathrm{B}\left(\theta_{\mathrm{BL}}\right)$ is the mean of values computed at sites $D, I, J$, and $P(1.11,1.11,0.97$, and $1.27 \mathrm{~m} / \mathrm{s}$, respectively). In this example, $\theta_{\mathrm{BL}}=1.12 \mathrm{~m} / \mathrm{s}$ is greater than $\theta_{\mathrm{AL}}$. This may reflect the fact that sites $U$ and $C$ are not precisely in the longitudinal direction. Effective transverse conduction velocity by method A $\left(\theta_{\mathrm{AT}}\right)$ was calculated using sites $T$ and $G\left(\theta_{\mathrm{AT}}=0.25 \mathrm{~m} / \mathrm{s}\right)$. This value is slightly lower than values that would have been obtained if $H$ and $B(\theta=0.27 \mathrm{~m} / \mathrm{s})$ or $\mathrm{S}$ and $\mathrm{F}(\theta=0.26 \mathrm{~m} / \mathrm{s})$ had been used. In the absence of impediments to transverse propagation, $\theta_{\mathrm{AT}}$ should be estimated by the lowest value normal to the activation front and perpendicular to fiber orientation because more rapid values may have elements of longitudinal conduction. Effective transverse conduction velocity by method B ( $\left.\theta_{\mathrm{BT}}\right)$ was obtained at sites $G$ and $N$ $\left(0.23\right.$ and 0.27 , respectively; $\left.\theta_{\mathrm{BT}}=0.25 \mathrm{~m} / \mathrm{s}\right)$. 


\section{Results}

Patients. The control group consisted of eight males and six females with a mean age ( \pm standard deviation) of $28 \pm 10 \mathrm{yr}$ (range 18-45) who underwent operation to interrupt an accessory atrioventricular connection. All had normal cardiac function as assessed by history, physical examination, and echocardiography. The patients with IDC consisted of 10 males and 5 females with a mean age of $40 \pm 14 \mathrm{yr}$ and a range $21-61 \mathrm{yr}$. All were undergoing cardiac transplantation for severe congestive heart failure but all were being managed as outpatients just before the time of transplantation. The mean ejection fraction was $0.14 \pm 0.05(0.10-0.23)$.

Determination of activation. In the presence of multiple low amplitude deflections, the test for activation provided a consistent method of assigning local activation. The test variable with the greatest ability to discriminate local and nonlocal signals was a linear combination variable $\psi=\ddot{\phi}_{3} / \phi_{30}$ $+0.0037 \phi_{30}$, where $\ddot{\phi}_{3}=$ the second derivative of the deflection and $\phi_{30}=$ the peak-to-nadir voltage of the deflection within $\pm 30 \mathrm{~ms}$ of $T_{\mathrm{D}}(53)$. The area under the ROC curve for $\psi(0.96)$ was significantly greater than the areas of the next best variables: $\ddot{\phi}_{3}(0.93, P=<0.001)$ and $\dot{\phi}_{\min }(0.88, P<0.001)$ (Fig. 3 $A)$. In addition, the maximum $Q_{\mathrm{E}}$ for $\psi(0.82)$ was significantly greater than those for $\ddot{\phi}_{3}\left(Q_{\mathrm{E}}=0.70, P=0.025\right)$ and $\dot{\phi}_{\min }\left(Q_{\mathrm{E}}\right.$ $=0.63, P=0.005)$. Plots of $Q_{\mathrm{E}}, Q_{\mathrm{SE}}$, and $Q_{\mathrm{SP}}$ as a function of $\psi$ are shown in Fig. $3 B$. By design, the threshold value of $\psi$ used to distinguish deflections resulting from local activation was that value $\left(\tau_{\mathrm{E}}\right)$ which maximized $Q_{\mathrm{E}}$. Thus, deflections with values of $\psi \geq 0.093$ were considered to be due to local activation and deflections with lesser values to be due to nonlocal activity. For this test $Q_{\mathrm{E}}=0.82, Q_{\mathrm{SE}}=0.70, Q_{\mathrm{SP}}=1.00$. The conventional statistics were: sensitivity $=0.89$, specificity $=1.00$, predictive value of a positive test $=1.00$, predictive value of a negative test $=0.79$. Application of the test for activation is illustrated in Fig. 4 for an electrogram with two deflections which have nearly equal minimum (greatest negative) first derivative ( $\dot{\phi}_{\min }$ ) values (Fig. $4 A$ ), for an electrogram with no deflections meeting criteria for activation (Fig. $4 B$ ), and for an electrogram with more than one deflection meeting criteria for activation (Fig. $4 \mathrm{D}$ ).

Activation patterns during constant cycle length stimulation. Spread of excitation in control subjects tended to be elliptical with relatively concentric isochrone contours. Activation patterns from an 18-yr-old woman undergoing operation for the Wolff-Parkinson-White syndrome are shown in Fig. 5. Rapid spread of activation was present where fiber orientation is perpendicular to the septum. The directions of rapid and slow propagation remained the same as the site of stimulation was changed. Electrograms recorded from the control patients were usually smooth with large intrinsic deflections and a dominant minimum in the first derivative plot (Fig. 5, $E$ and $F$ ). These features are consistent with uniform anisotropic propagation and were observed in 12 out of the 14 control subjects $(86 \%)$. In these 12 patients $\theta_{\mathrm{AL}}=0.80 \pm 0.08 \mathrm{~m} / \mathrm{s}, \theta_{\mathrm{AT}}$ $=0.23 \pm 0.03 \mathrm{~m} / \mathrm{s}$ and $\theta_{\mathrm{AL}} / \theta_{\mathrm{AT}}=3.5 \pm 0.6$. The second method of estimating conduction velocity produced similar values: $\theta_{\mathrm{BL}}$ $=0.79 \pm 0.11 \mathrm{~m} / \mathrm{s}, \theta_{\mathrm{BT}}=0.23 \pm 0.04 \mathrm{~m} / \mathrm{s}$ and $\theta_{\mathrm{BL}} / \theta_{\mathrm{BT}}=3.3 \pm 0.3$. Two of the control patients had moderately disturbed activation patterns similar to those present in group II IDC patients described below.

The patients with IDC were extremely heterogeneous with respect to the patterns of activation that were recorded. They
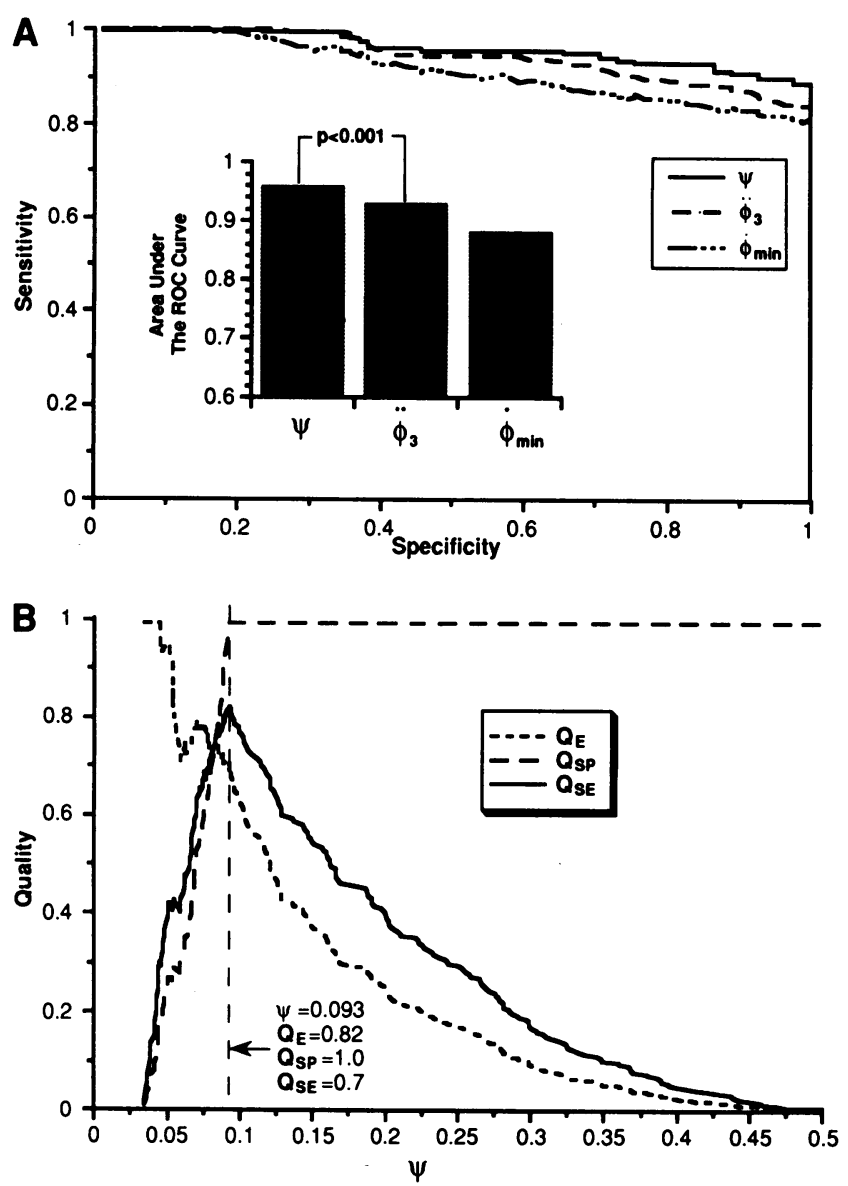

Figure 3. (A) ROC curves for the three candidate variables for tests for activation with the greatest power to discriminate local from distant electrical activity: $\psi=\ddot{\phi}_{3} / \phi_{30}+0.0037 \phi_{30}$, the second derivative $\left(\ddot{\phi}_{3}\right)$, and the minimum first derivative $\left(\dot{\phi}_{\min }\right)$. The area under the ROC curve, a measure of test performance, was significantly higher for $\psi$ (inset bar graph). (B) Plot of $\psi$ versus the "quality" statistics: the quality of efficiency $\left(Q_{\mathrm{E}}\right)$, the quality of specificity $\left(Q_{\mathrm{SP}}\right)$, and the quality of sensitivity $\left(Q_{\mathrm{SE}}\right)$. The value of $\psi$ which maximized $Q_{\mathrm{E}}$ was $\tau_{\mathrm{E}}=0.093$ (dashed vertical line).

could be divided into three groups. Group I consisted of 7 out of the 15 patients with IDC (47\%) with uniform anisotropic propagation, i.e., with smooth electrograms and few irregularities in the activation patterns (Fig. 2). Although there was a slight tendency toward greater $\theta_{\mathrm{L}}$ and higher $\theta_{\mathrm{L}} / \theta_{\mathrm{T}}$ in these IDC patients, the propagation velocities did not differ significantly from the values in control patients: $\theta_{\mathrm{AL}}=0.84 \pm 0.09 \mathrm{~m} / \mathrm{s}, \theta_{\mathrm{AT}}$ $=0.23 \pm 0.05 \mathrm{~m} / \mathrm{s}$ and $\theta_{\mathrm{AL}} / \theta_{\mathrm{AT}}=3.7 \pm 0.7\left(\theta_{\mathrm{BL}}=0.83 \pm 0.08\right.$ $\left.\mathrm{m} / \mathrm{s}, \theta_{\mathrm{BT}}=0.23 \pm 0.04 \mathrm{~m} / \mathrm{s}, \theta_{\mathrm{BL}} / \theta_{\mathrm{BT}}=3.7 \pm 0.7\right)$. Therefore, propagation in group I were consistent with pattern A (i.e., no difference from controls) described in Methods. While it could be argued that a tendency for pattern $\mathrm{C} 2$ was observed, i.e., increased $\theta_{\mathrm{L}}$ and $\theta_{\mathrm{L}} / \theta_{\mathrm{T}}$ with unchanged $\theta_{\mathrm{T}}$, patterns $\mathrm{B}, \mathrm{D}, \mathrm{E}$, and $F$ were clearly absent.

Group II comprised four patients with IDC (27\%) who had moderately disturbed activation patterns. An example from a 35-yr-old man with IDC is shown in Fig. 6 A. Regions of dense isochrome spacing are present in the right lower and in the left upper corners of the map. It is not possible to determine whether the areas of dense isochrone contours resulted from slow conduction or from conduction block. It is evident, how- 


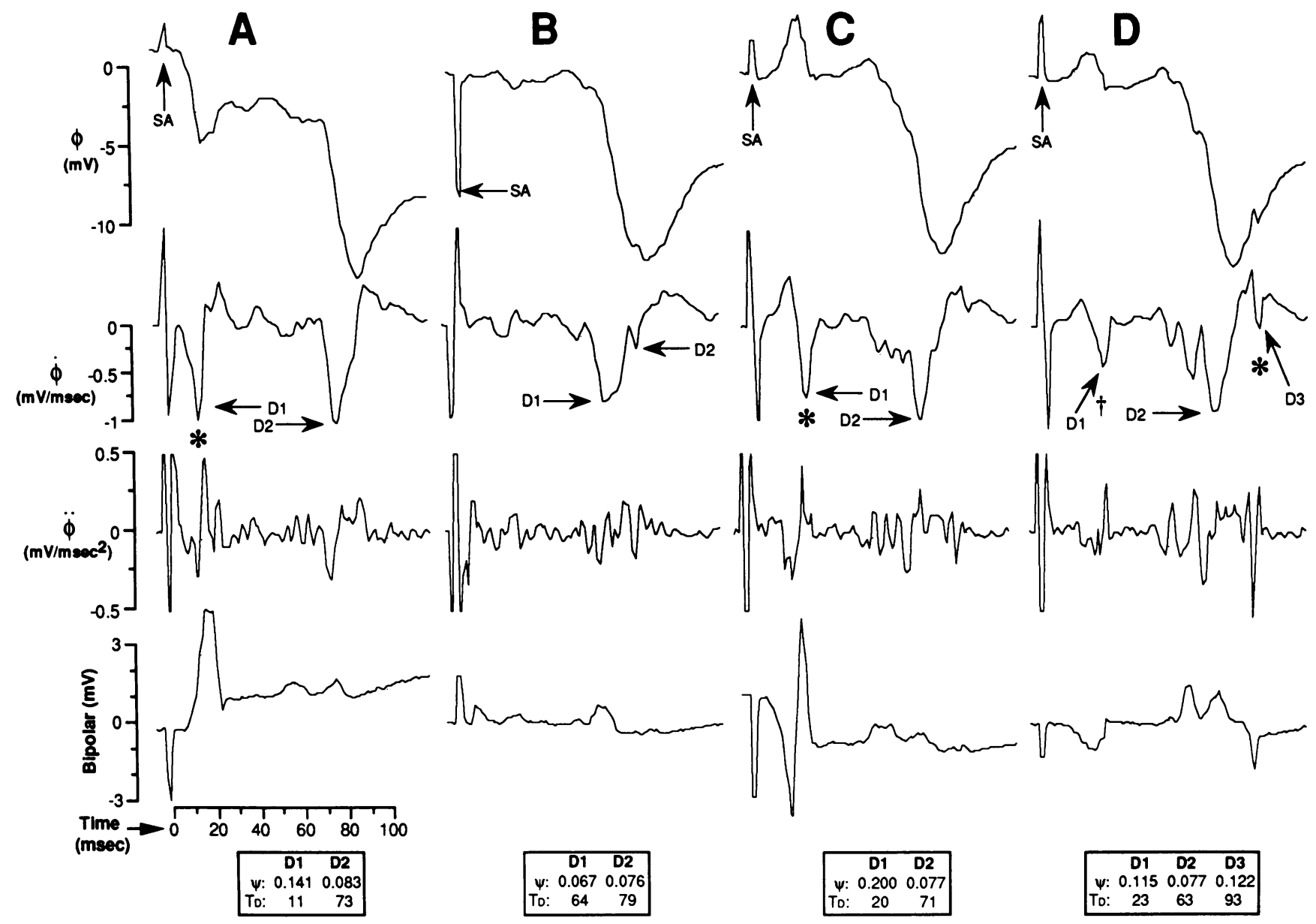

Figure 4. Polyphasic recordings obtained from a patient with IDC. The top row of tracings is the unipolar extracellular potential ( $\phi)$, the second row is the first derivative of $\phi(\dot{\phi})$, and the third row is the second derivative of $\phi(\ddot{\phi})$. The fourth row is a bipolar signal obtained by subtracting the unipolar signal shown in the top row, from the unipolar signal of its neighbor. This is equivalent to the signal obtained from a pair of electrodes $2 \mathrm{~mm}$ apart. The deflections are labeled in the plot of $\dot{\phi}(D 1, D 2$, etc.) where they are most easily recognized. For each deflection, the value of the linear combination variable $(\psi)$ and the time of the deflection $\left(T_{\mathrm{D}}\right)$ with respect to the stimulus artifact $(S A)$ are given in the table below each column of plots. An asterisk (*) indicates the deflection in each electrogram with the greatest value of $\psi$ which exceeds the threshold $\tau_{\mathrm{E}}=0.093$, a dagger $(\dagger)$ indicates other deflections that exceed $\tau_{\mathrm{E}} .(A)$ The two prominent deflections $D 1$ and $D 2$ have nearly equal values of the minimum first derivative $\left(\dot{\phi}_{\min }\right)$, but $D I$ has a greater peak-to-nadir second derivative value within \pm 3 msec $\left(\ddot{\phi}_{3}\right)$. Because the test variable $(\psi$ $\left.=\ddot{\phi}_{3} / \phi_{30}+0.0037 \phi_{30}\right)$ is, in essence, $\ddot{\phi}_{3}$ normalized by the voltage of the signal, its value for $D 1$ is greater than for D2. Thus, Dl meets criteria for activation whereas $D 2$ does not. The more prominent bipolar deflection corresponding to D1 supports this. $(B)$ None of the deflections in this signal achieve the threshold for $\psi$ : Local activation is considered absent at this site. $(C) D 1$ meets activation criteria despite a low $\phi$ and lower $\dot{\phi}_{\min }$ than D2. The bipolar signal supports this choice. $(D)$ The change in $\phi$ associated with $D 3$ is obscured by the rising phase of a large, slow potential caused by distant electrical activity, but the sharp $\dot{\phi}$ and relatively large $\ddot{\phi}$ reveal its high frequency content and account for the relatively large value of $\psi . D 1$ also meets activation criteria thus, this site would be considered to have two activation times. Multiple activation times at a single site have been reported previously $(49,50)$.

ever, that the areas with activation delays do not consist entirely of nonconducting tissue. In the upper left corner of Fig. 6 $A$, for instance, propagation through the sites labeled 1,2 , and 3 appears normal. However, stimulation from the lower edge of the plaque resulted in delayed activation through this same area (Fig. $6 \mathrm{~B}$ ). The direction of propagation also affected the configuration of the electrograms. Fig. $6 C$ shows $\phi$ and $\dot{\phi}$ recorded during longitudinal propagation at the sites labeled 1,2 , and 3 in Fig. $6 \mathrm{~A}$. The intrinsic deflections of the electrograms are smooth and produce derivatives with a single prominent deflection. The amplitudes of $\phi$ and $\dot{\phi}_{\min }$ are similar to those from control subjects (Fig. 5). The amplitudes of $\phi$ and $\dot{\phi}_{\min }$ during transverse propagation at the same sites (Fig. $6 \mathrm{D}$ ), however, are much smaller and have multiple low amplitude deflections (fractionation) $(50,61)$. This combination of features is consistent with nonuniform anisotropic properties (pattern E).

The effective longitudinal conduction velocities in the four IDC patients with moderately disturbed activation patterns tended to be high $\left(\theta_{\mathrm{AL}}=0.90 \pm 0.09 \mathrm{~m} / \mathrm{s}, \theta_{\mathrm{BL}}=0.88 \pm 0.07\right.$ $\mathrm{m} / \mathrm{s}$ ), but were not significantly higher than control subjects with uniform activation patterns. The marked alterations in transverse propagation precluded reliable estimation of transverse conduction velocity.

The four patients with IDC in group III had "severely" disturbed activation patterns. Group III patients differed from group II patients in that both transverse and longitudinal propagation appeared to be disturbed, and group III patients tended 

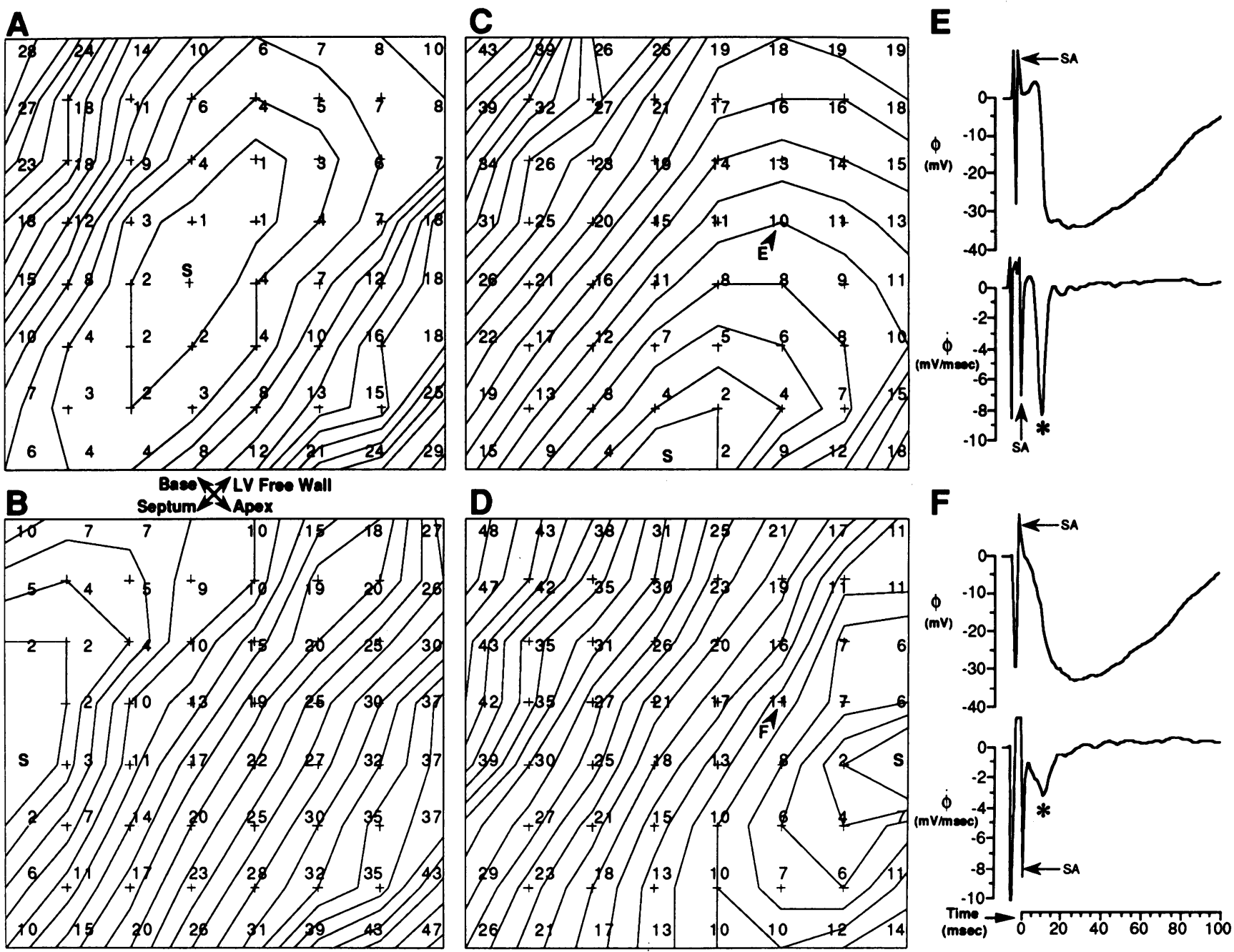

Figure 5. $(A-D)$ Electrical activation sequence maps based on recordings from a control subject; same format as Fig. $2 . S=$ site of stimulation; isochrone contours are drawn at 2-msec intervals. The labeled electrode sites correspond to the electrograms shown in panels $E$ and $F$. ( $E$ ) Extracellular potential $(\phi)$ and first temporal derivative $(\dot{\phi})$ recorded at the electrode site marked $E$ in panel $C$ during propagation in the longitudinal direction. $\phi$ consists of a large, smooth intrinsic deflection preceded by a small $\mathrm{R}$ wave. The peak to peak amplitude is $38 \mathrm{mV}$. The first derivative consists of a single predominant minimum $\left(\dot{\phi}_{\min }=-8.3 \mathrm{mV} / \mathrm{msec}\right)$. $(F)$ Recording obtained when the electrode site was in the transverse direction (panel $D$ ). The peak-to-nadir amplitude of $\phi$ is $33 \mathrm{mV}, \dot{\phi}_{\min }=-3.1 \mathrm{mV} / \mathrm{msec}$. The changes in extracellular waveforms during propagation in different directions were consistent with previous descriptions in other species (35). $S A$, stimulus artifact.

to have larger differences of activation times between neighboring recording sites. Isochrone maps based on recordings from a group III patients is shown in Fig. 7, samples of electrograms upon which the maps are based are shown in Figs. 4, 8, and 9. The maps are characterized by regions of closely spaced isochrone contours resulting from large differences in activation times between adjacent electrode sites (e.g., $73 \mathrm{~ms}$ between sites $C$ and $D$ in Fig. $7 A$, which correspond to the electrograms in Fig. 4, $C$ and $D$, and $117 \mathrm{~ms}$ between sites $A$ and $B$ in Fig. $7 C$, which correspond to the electrograms in Fig. 9, $A$ and $B$ ). The activation path that accounts for the large gradients in activation times cannot be determined with precision. However, based on previous work (56), the presence of near simultaneous activation times distal to the band of dense isochrones (i.e., the lower halves of the maps in Fig. 7, $A$ and $C$ ) suggest that activation spreads to this area from an intramural route. On the other hand, very slow conduction through the regions of dense isochrones cannot be excluded. There are also areas lacking evidence of local electrical activity, e.g., site $B$ in Fig. $7 A$, which corresponds to the electrogram in Fig. $4 B$.

The test or criteria used to define activation can obviously affect the pattern of activation especially in the presence of multiple low amplitude deflections $(53,61)$. The test for activation used to select activation times was established before activation maps were constructed so that the choices for activation times would not be influenced by preconceived notions about activation sequences. However, it is important to assess the impact of a particular test for activation on the observed activation patterns which can be done by adjusting the test threshold $\tau$. Reducing $\tau$ to 0.070 results in a more sensitive but less specific test for activation with $Q_{\mathrm{SP}}=0.50, Q_{\mathrm{SE}}=0.78, Q_{\mathrm{E}}$ $=0.61$, conventional sensitivity $=0.95$, and conventional specificity $=0.60$ (compare to statistics for $\tau=0.093$ given above) . The effect of using this more sensitive, less specific test for activation (Fig. 7, $B$ and $D$ ) is to "activate" the "nonactivated" areas in Fig. 7, $A$ and $B$ and to increase the number of sites with 

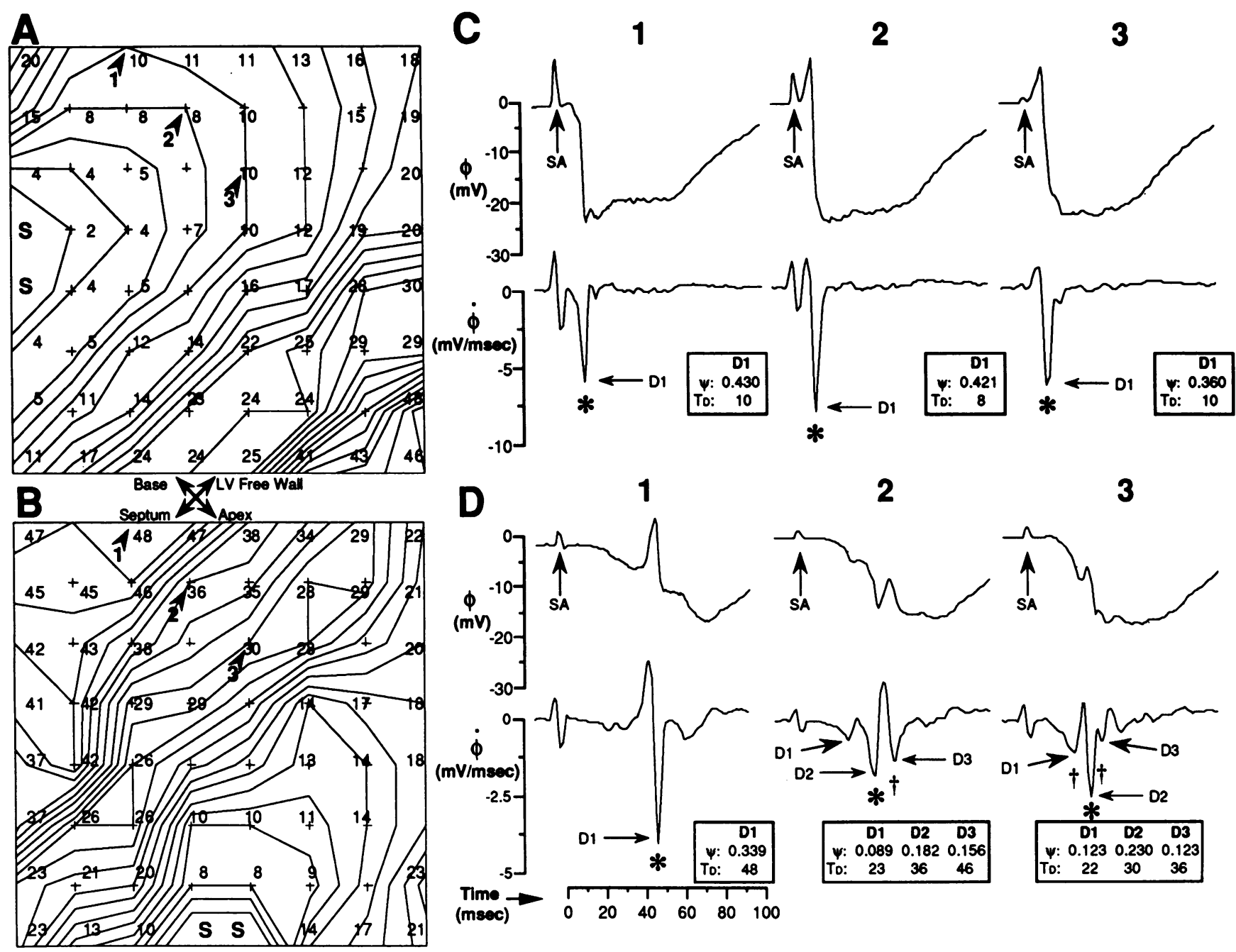

Figure 6. Electrical activation sequences from a 35-yr-old man with IDC. The interval between isochrones is 2 msec. The orientation of the array is shown in the diagram between the two maps. $(A)$ Isochrone map during stimulation at the left edge of the array $(S)$ shows rapid activation parallel to the long axes of myocardial fibers. Propagation transverse to fiber orientation is abnormal, with areas of delayed activation (dense isochrones) especially in the lower left lower corner. Propagation across sites labeled 1,2 , and 3 appears normal. (B) Isochrone map obtained during stimulation at the lower edge of the array. The directions of rapid and slow propagation are unchanged from the map in panel $A$, but the regions with slow activation have changed. Activation across sites labeled 1,2 , and 3 now appears abnormally slow. $(C)$ Unipolar potentials $(\phi$, top row) and first derivative plots ( $\dot{\phi}$, bottom row) recorded at sites labeled 1,2 , and 3 in panel $A$. The values of $\psi$ and the times of the deflections $\left(T_{\mathrm{D}}, \mathrm{msec}\right)$ are given in the insets. $(D) \phi$ and $\dot{\phi}$ plots recorded at sites 1,2 , and 3 in panel $B$. In contrast to the signals recorded during longitudinal propagation at the same locations (panel $C$ ), the signals recorded during transverse propagation exhibit multiple deflections with lower amplitudes. The two deflections in electrode $2(D 1$ and $D 2$ ) both meet criteria for activation, i.e., both have values of $\psi$ greater than the test threshold $\left(\tau_{E}=0.093\right)$. This suggests asynchronous activation in adjacent muscle bundles $(50)$. By convention, the isochrone maps were drawn according to the deflection with the greatest value of $\psi$. Altering the choice of the deflection representing local activation would alter the location of the densely spaced isochrones; e.g., if $D 3$ of electrode 2 in panel $D$ had been chosen to represent local activation, the group of dense isochrones would switch from between electrodes 1 and 2 to between electrodes 2 and 3. This effect has been illustrated previously by Dillon et al. (61), but, as they have discussed, switching the deflection designated as local activation does not alter the finding of disturbed transverse propagation.

multiple activation times. However, the large transitions in activation times and the severity of the conduction disturbances are not affected.

Effects of premature stimulation on activation sequence. The effects of premature beats on activation sequence are of interest because spontaneous premature beats may initiate arrhythmias by altering myocardial propagation $(43,61,62)$. Eight ventricular stimuli were delivered at a cycle length of 400 or $500 \mathrm{~ms}$ followed by up to three premature stimuli with coupling intervals within $10 \mathrm{~ms}$ of the refractory period of the preceding beat. In the four control subjects in whom this proto- col was performed, slight changes in activation patterns were sometimes observed (Fig. 10), but at most sites the activation times did not change by more than a few milliseconds, and longitudinal and transverse conduction velocities changed only slightly. Also, the total activation time (TAT), defined as the latest activation time minus the earliest activation time, changed little with premature stimuli. This suggests that with the stimulus strength used (twice diastolic threshold current) the premature beats could not be delivered early enough in the cell cycle to severely limit sodium channel current. The responses of $\theta_{\mathrm{AL}}$ and TAT to premature stimuli are plotted for 

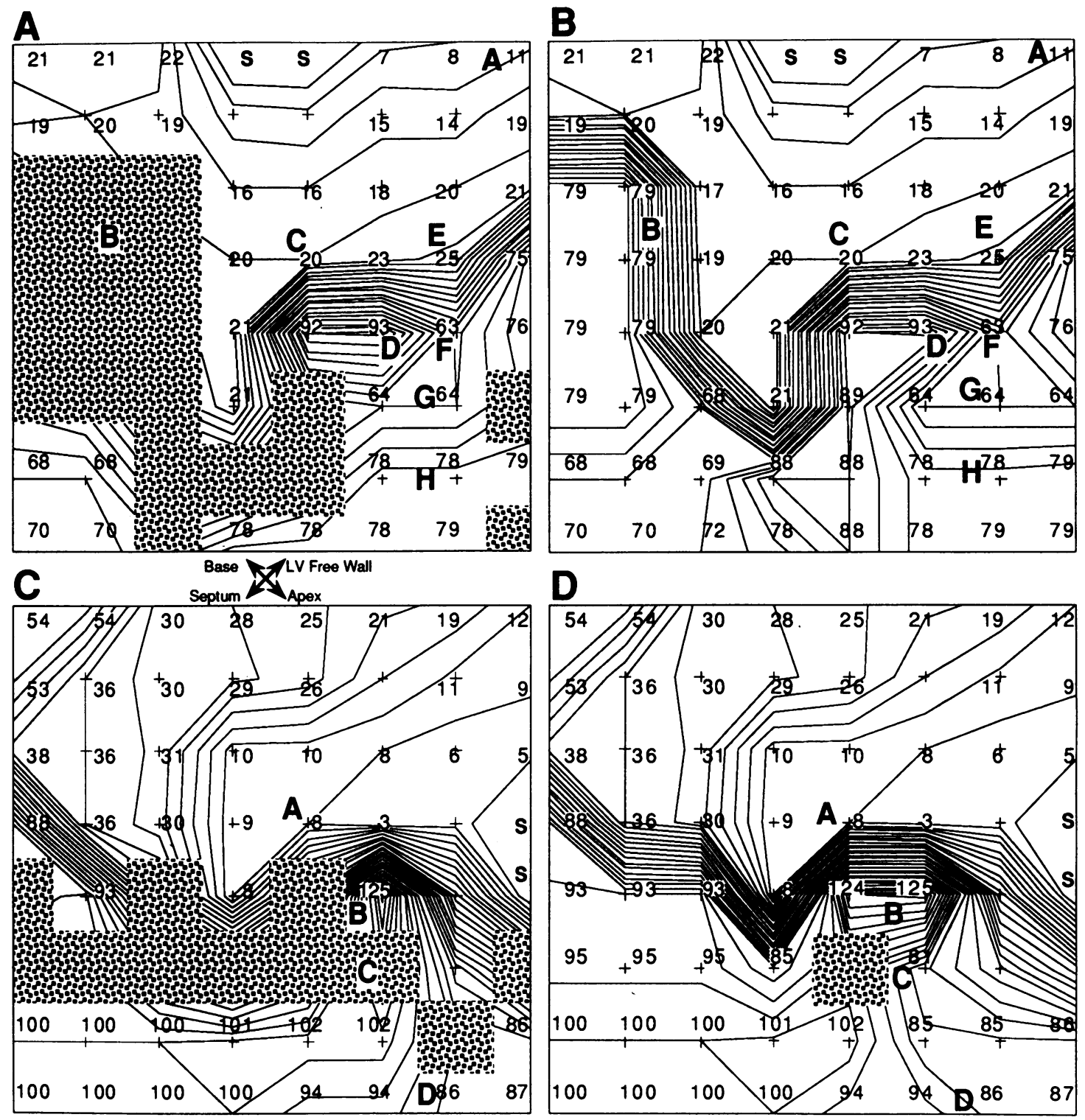

Figure 7. Electrical activation maps from a 35-yr-old woman with IDC. The format is the same as in Fig. 2 except that the interval between contours is $4 \mathrm{~ms}$. The stippled regions include electrode locations at which no deflection met criteria for local activation. $(A)$ Activation sequence during stimulation from the upper edge $(S)$. The presence of activation was based on the test threshold $\tau_{\mathrm{E}}=0.093$. Electrograms from sites $A$, $B, C$, and $D$ are shown in Fig. $4, A, B, C$, and $D$, respectively. Electrograms from sites $E, F, G$, and $H$ are shown in Fig. 8, $E, F, G$, and $H$, respectively. ( $B$ ) Map based on the same electrograms as the map in panel $A$ but with presence of local activation defined by $\tau=0.070$. $(C)$ Activation sequence during stimulation from the right edge $(S)$. The presence of activation was based on the test threshold $\tau_{\mathrm{E}}=0.093$. Electrograms from sites $A, B, C$, and $D$ are shown in Fig. 9, $A, B, C$, and $D$, respectively. (D) Map based on the same electrograms as the map in panel $C$ but with presence of local activation defined by $\tau=0.070$.

individual patients in Fig. 11. There was a consistent tendency for reduction of $\theta_{\mathrm{AL}}$ in the patients with IDC with $V_{2}$ and $V_{4}$, although $V_{3}$ tended to result in less depression of $\theta_{\mathrm{AL}}$. These findings were not changed when method $B$ was used to estimate $\theta_{\mathrm{L}}$. For reasons discussed earlier, $\theta_{\mathrm{T}}$ could not be reliably estimated in many of the patients with IDC. TAT could be compared in all patients in whom premature stimuli were administered including two patients with IDC in whom activation was too distorted to determine $\theta_{\mathbf{L}}$. There was a clear increase in TAT in some patients with IDC, again suggesting heterogeneity in the response to premature stimulation in patients with this disorder.
Although the average effects of premature stimulation were not dramatic in the patients with IDC as a group, striking changes were noted in some individuals. The maps in Fig. 12 were obtained with a train of eight stimuli $\left(S_{1}\right)$ at a cycle length of $500 \mathrm{~ms}$ followed by three premature stimuli $\left(S_{2}, S_{3}, S_{4}\right)$ at intervals of 260,230 , and $210 \mathrm{~ms}$. $S_{4}$ did not capture the ventricles but was followed by two ectopic beats $\left(V_{4}\right.$ and $\left.V_{5}\right)$. The clearest indication of this is the reversal of activation order at sites $A, B$, and $C$ in Fig. 12 (the electrograms recorded at these sites are shown in Fig. 13). Although site of origin of the ectopic beats cannot be precisely identified, two features suggest it was relatively close to the tissue in contact with the electrode 


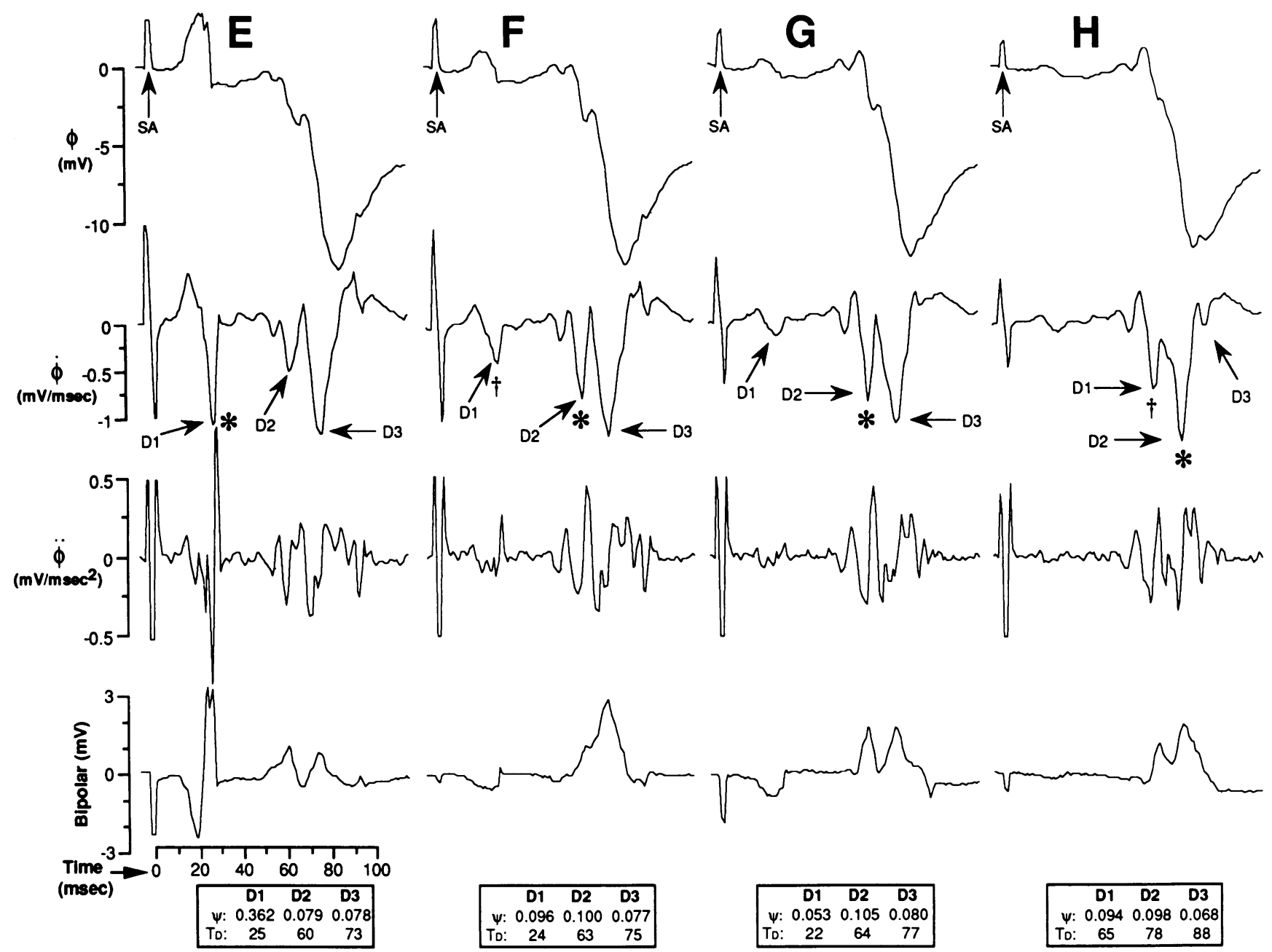

Figure 8. Electrograms obtained from the electrode sites labeled $E, F, G$, and $H$ in Fig. 7, $A$ and $B$. Same format as Fig. 4. $(E)$ Activation at this site occurs early $(D 1$ at $25 \mathrm{msec})$ but deflections due to distant activity are apparent at 60 and 73 msec. $(F)$ Two deflections $(D 1$ and $D 2)$ meet criteria for activation with marginal values of $\psi$. This suggests that the electrode straddles two or more poorly coupled myocardial cell bundles. ( $G$ and $H$ ) The deflections meeting activation criteria occur relatively late in these leads demonstrating a progression of activation times across the region of dense isochrones present in Fig. $7 \mathrm{~A}$.

array. The first is that during the ectopic beats, sites depicted in the upper right corner and center of the map were activated early compared to sites in the lower half and the left upper corner which were activated much later. If the site of origin were distant from the plaque array, a pattern closer to that observed during sinus rhythm (Fig. $12 F$ ) would be expected, that is, a pattern with much less dispersion of activation times. In fact during the sinus beat, where activation of the tissue beneath the plaque presumably spread from the endocardium, central sites were activated later than peripheral sites. Second, it has been shown that a short time interval between the onset of the root mean square (RMS) voltage curve and the site of earliest activation ( $5 \mathrm{~ms}$ for $V_{4}$ and $V_{5}$ ) indicates proximity of the origin of electrical activity and the site of earliest recorded activation (63). In contrast, earliest recorded activation occurred 44 ms after the onset of the RMS voltage for a sinus beat (Fig. $12 F$ ).

Of additional interest is the marked conduction delay that developed with premature beats. During a sinus beat, the region in the left upper corner of the maps in Fig. 12 is activated early compared to most recorded sites (Fig. $12 \mathrm{~F}$ ). During $V_{1}$, activation in the left upper corner is relatively late, e.g., $47 \mathrm{~ms}$ at site $\mathrm{F}$ (Fig. $12 \mathrm{~A}$ ). With each successive premature beat the activation time of this region increases, e.g., at site $F 74 \mathrm{~ms}$ during $V_{2}, 119 \mathrm{~ms}$ during $V_{3}, 166 \mathrm{~ms}$ during $V_{4}$, and $176 \mathrm{~ms}$ during $V_{5}$. Although $V_{4}$ and $V_{5}$ are ectopic beats, activation of the central sites occurs early, and only about $10 \mathrm{~ms}$ of the delay observed at site $F$ could be attributed to the change in activation sequence associated with the ectopic beats.

Of further possible significance is the finding that large gradients of activation time occurred over very short distances. Fig. $12 E$ shows, for instance, a difference in activation times of $164 \mathrm{~ms}$ between sites $D$ and $E$, which were only $2.8 \mathrm{~mm}$ apart. The electrograms upon which these activation times were based are shown in Fig. 14. In particular, the electrograms from site $E$ (Fig. $14 E$ ) demonstrate progressive activation delays between primary deflections (those with the highest values of $\psi$ ) and secondary deflections (those which meet activation criteria but have lesser values of $\psi$ ). Again, the presence of multiple deflections which meet activation criteria suggests that the recording site straddled multiple muscle bundles. If true, these observations indicate that large activation delays can occur be- 


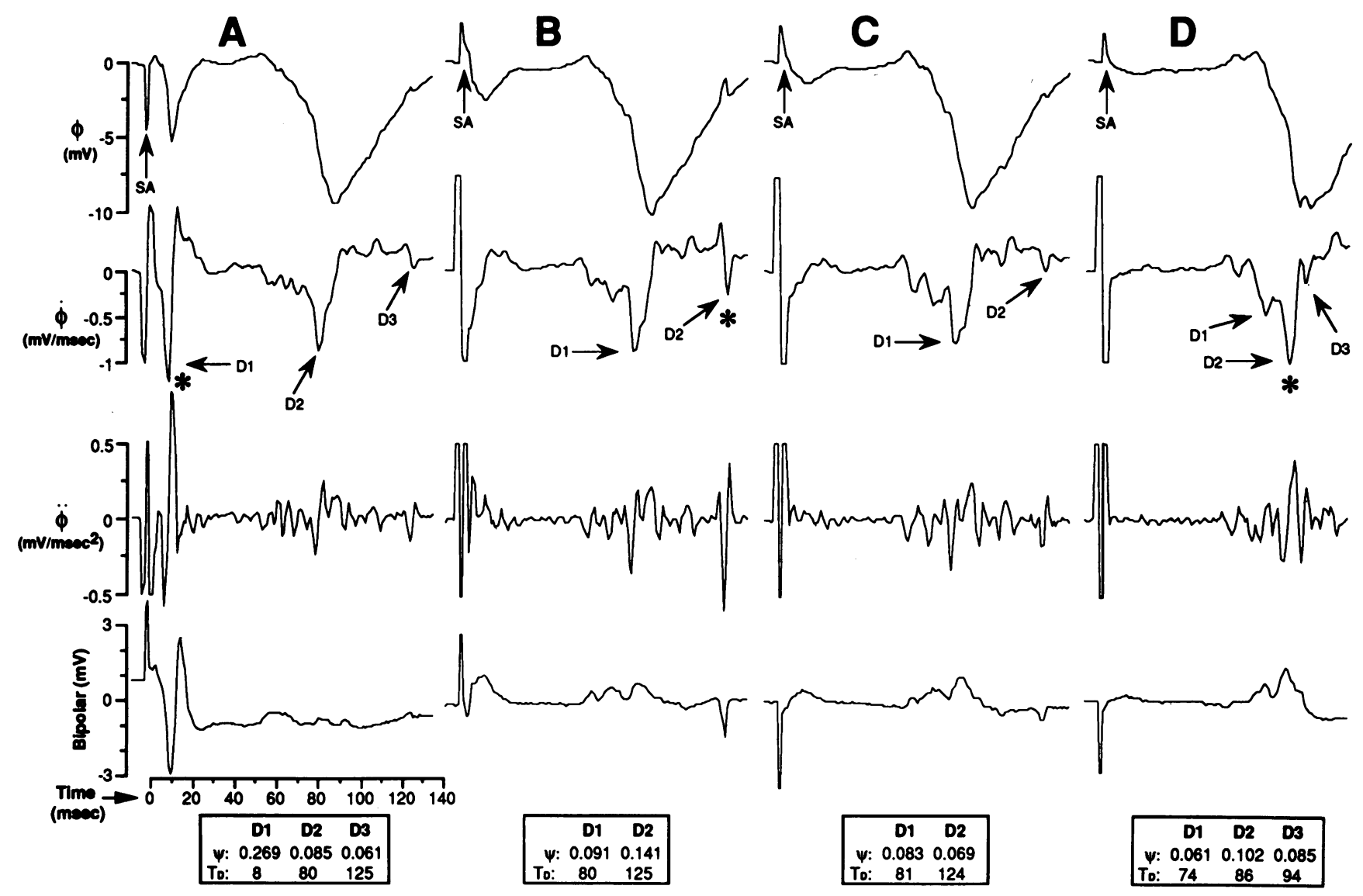

Figure 9. Electrograms obtained from the electrode sites labeled $A, B, C$, and $D$ in Fig. 7, $C$ and $D$. Same format as Fig. 4. ( $A$ ) The deflection labeled $D 1$ has a value of $\psi$ well above threshold for activation and a prominent bipolar signal. Later deflections are present at 80 and 125 msec, but do not attain activation criteria and do not produce a discernable bipolar deflection indicating that $D 2$ and $D 3$ are probably due to distant activity. $(B)$ This electrogram recorded from site $B$ in Fig. $7 C$ includes a deflection that meets activation criteria that occurs remarkably late compared to the activation of the neighboring site $A$. $(C)$ None of the deflections meet criteria for activation in this electrogram with the threshold $\tau=0.093$. Lack of activation at this site suggests poor conduction and could indicate an insulating effect for the tissue responsible for the very late activation time at site $B .(D)$ The deflection meeting activation criteria $(D 2)$ occurs much later than activation at site $A$, but is earlier than that at site $B$ despite its more distal location from the stimulation site. This indicates delayed conduction between sites $D$ and $B$ and the relatively protected status of viable tissue at site $B$.

tween bundles of myocardial fibers muscle bundles $<1 \mathrm{~mm}$ apart. It is noteworthy that these conduction delays were produced by premature beats with coupling intervals similar to those which had minimal effects on conduction velocities and activation patterns in controls and IDC patients with uniform anisotropic properties. Finally, the association between ectopic beats induced by ventricular extrastimuli and the development of large activation delays raises the possibility that the induced ectopic beats resulted from reentrant excitation.

$N A I$. The NAI is measure of the spatial heterogeneity of activation times, and has been used to quantify the degree of nonuniformity of activation (56). The patients with IDC had significantly greater NAIs $(9.1 \pm 6.1 \mathrm{~ms} / \mathrm{mm})$ than the control patients $(5.5 \pm 1.4 \mathrm{~ms} / \mathrm{mm}, P=0.025)$. The degree of disturbance of the activation patterns corresponded with the NAIs: group I had a mean NAI of $6.0 \pm 1.3 \mathrm{~ms} / \mathrm{mm}$, group II had a mean NAI of $9.6 \pm 2.2 \mathrm{~ms} / \mathrm{mm}$, and group III had a mean NAI of $13.8 \pm 10.7 \mathrm{~ms} / \mathrm{mm}$.

Histology. Myocardial tissue from beneath the recording plaque was examined in 13 of the 15 patients with IDC. The mean percentage of fibrous tissue was $13.5 \pm 15.8 \%$ which is similar to that reported previously $(10,15,18,19,20,23)$. Patients in group I had minimal fibrosis with a mean fraction of only $4.6 \pm 5.6 \%$ (Fig. 15, top), group II patients had intermediate amounts of fibrosis: $9.5 \pm 7.8 \%$ (Fig. 15 , middle), and patients in group III had very prominent fibrosis: $28.3 \pm 21 \%$ (Fig. 15 , bottom $)$. There was a significant correlation between the NAI and the fraction of fibrosis $(r=0.57, P<0.05)$. Moreover, the pattern of fibrosis in the groups with mild and moderate disturbances of activation patterns was interstitial, that is, primarily linear accumulations of collagen separating bundles of myocytes with little alteration in the alignment of myocytes or bundles. In contrast, replacement fibrosis and microscopic scars were prominent in group III patients which often was accompanied by deviation or interruption of the parallel arrangement of epicardial muscle fibers and bundles (16).

Relation between electrophysiologic findings and spontaneous arrhythmias. 12 of the control patients underwent at least $24 \mathrm{~h}$ of electrocardiographic monitoring (mean $=63 \pm 33 \mathrm{~h}$ ); no ventricular arrhythmias were detected in nine of these patients. In two of the control patients rare pairs of premature ventricular contractions (PVCs) were recorded. Only one con- 
A

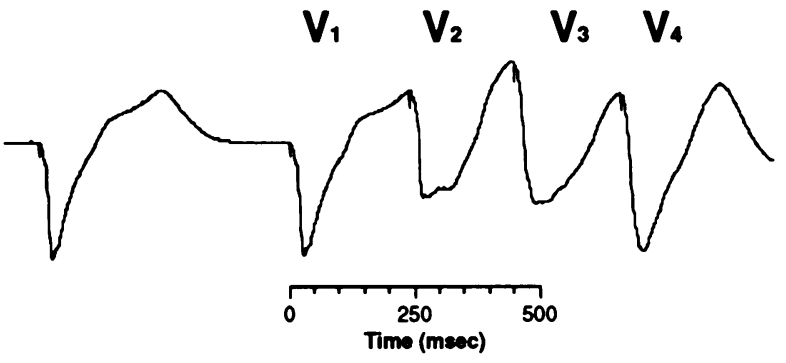

$V_{1}=500, V_{2}=240, V_{3}=210, V_{4}=210 \mathrm{msec}$
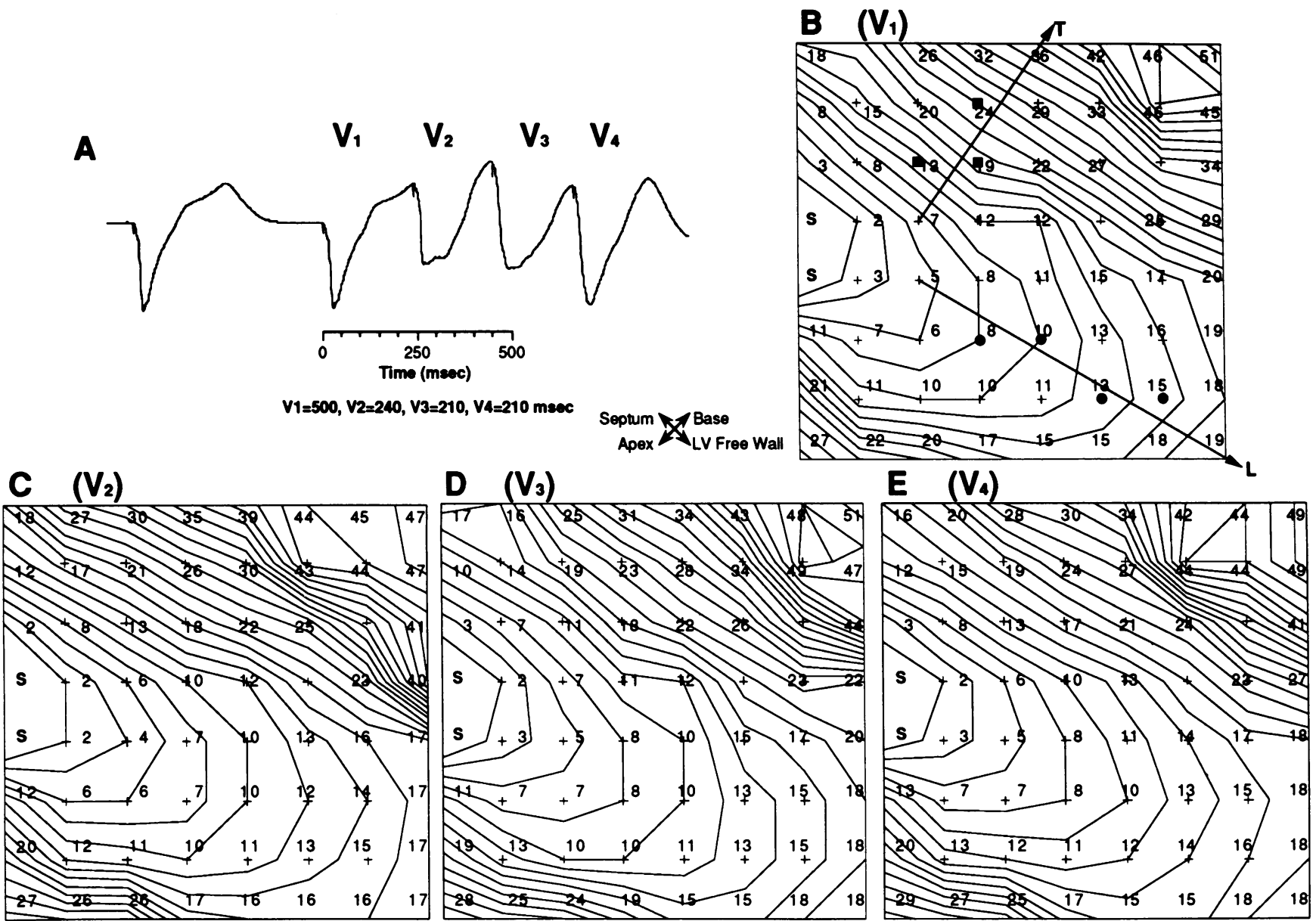

Figure 10. Effects of premature stimulation in a 35-yr-old male control subject. $(A)$ An electrogram from the recording site in the upper left corner of the array shows the last two beats in the eight beat train $\left(V_{1}\right)$, and the first $\left(V_{2}\right)$, second $\left(V_{3}\right)$, and third $\left(V_{4}\right)$ premature stimuli. The cycle length of the train of stimuli was $500 \mathrm{msec}$, and the coupling intervals of $V_{2}, V_{3}$ and $V_{4}$ were 250,210, and $210 \mathrm{msec}$, respectively. (B) Map of $V_{1}$. The format is the same as in Fig. 2. Stimulation is performed from the left edge $(S)$, the interval between isochrones is 2 msec. The arrows indicate the directions of longitudinal $(L)$ and transverse $(T)$ propagation. The electrode sites indicated by the heads and tails of the arrows were used to estimate longitudinal and transverse conduction velocities by method $\mathrm{A}\left(\theta_{\mathrm{AL}}, \theta_{\mathrm{AT}}\right)$ The electrode sites indicated were used to estimate longitudinal $(\bullet)$ and transverse $(\bullet)$ conduction velocities by method $\mathrm{B}\left(\theta_{\mathrm{BL}}, \theta_{\mathrm{BT}}\right)$. In this example, $\theta_{\mathrm{AL}}=\theta_{\mathrm{BL}}=0.83 \mathrm{~m} / \mathrm{sec}$ and $\theta_{\mathrm{AT}}$ $=\theta_{\mathrm{BT}}=0.25 \mathrm{~m} / \mathrm{sec}$. The TAT is $49 \mathrm{msec}$. $(C)$ Map of $V_{2}: \theta_{\mathrm{AL}}=0.90 \mathrm{~m} / \mathrm{sec}, \theta_{\mathrm{BL}}=0.80 \mathrm{~m} / \mathrm{sec}, \theta_{\mathrm{AT}}=\theta_{\mathrm{BT}}=0.22 \mathrm{~m} / \mathrm{sec}, \mathrm{TAT}=45 \mathrm{msec}$. $(D)$ Map of $V_{3}: \theta_{\mathrm{AL}}=0.90 \mathrm{~m} / \mathrm{sec}, \theta_{\mathrm{BL}}=0.87 \mathrm{~m} / \mathrm{sec}, \theta_{\mathrm{AT}}=0.27 \mathrm{~m} / \mathrm{sec}, \theta_{\mathrm{BT}}=0.25 \mathrm{~m} / \mathrm{sec}$, TAT $=49 \mathrm{msec} .(E) \mathrm{Map} \mathrm{of} \mathrm{V}_{4}: \theta_{\mathrm{AL}}=0.90 \mathrm{~m} / \mathrm{sec}, \theta_{\mathrm{BL}}$ $=0.86 \mathrm{~m} / \mathrm{sec}, \theta_{\mathrm{AT}}=0.26 \mathrm{~m} / \mathrm{sec} \theta_{\mathrm{BT}}=0.25 \mathrm{~m} / \mathrm{sec}$, TAT $=47 \mathrm{msec}$.

trol subject had frequent ventricular arrhythmias (265 PVCs/ h, 0.1 pairs $/ \mathrm{h}$ ). Interestingly, this was one of two control patients with moderately altered activation. 10 of the 15 patients with IDC underwent 24-h Holter monitoring, five in group I, four in group II, and one in group III. Because of the small numbers, groups II and III were combined. The PVC frequency in the combined group was $478 \pm 678 \mathrm{PVCs} / \mathrm{h}$ (median $=121 \mathrm{PVCs} / \mathrm{h})$ compared with group I patients who had a mean of $123 \pm 240 \mathrm{PVCs} / \mathrm{h}$ (median: $21 \mathrm{PVCs} / \mathrm{h})(P=0.1)$. The frequency of PVC pairs in the combined group was 25 pairs $/ \mathrm{h}($ median $=2$ pairs $/ \mathrm{h}$ ) compared to $16 \pm 25$ pairs $/ \mathrm{h}$ ( median $=0.3$ pairs $/ \mathrm{h})(P=0.26)$. The frequency of unsustained ventricular tachycardia (VT) in the combined group was $8 \pm 11 / \mathrm{h}($ median $=6.2 / \mathrm{h})$ compared to only $0.8 \pm 1.7 / \mathrm{h}(\mathrm{me}-$ dian $=0)$ in the group I patients $(P=0.17)$. In addition, all of the patients in the combined group had PVC frequencies $>30 /$ $h$ and all but one had unsustained VT during the 24-h Holter recording, whereas only one of the group I patients had $>30$
PVCs/h, and only two had an episode of unsustained VT. Thus, patients in groups II and III tended to have greater frequencies of each category of arrhythmia, but none of the differences attained statistical significance. On the other hand, it should be recognized that there were relatively few patients in each group, the variances were high, and there was no data from three of the four group III patients who might have been expected to have the most severe ventricular arrhythmias. Only one of the seven group I patients had a history of sustained ventricular tachycardia (lasting at least 1 min or requiring cardioversion), which occurred during an infusion of dopexamine, a $\beta$-adrenergic and dopaminergic receptor agonist. In contrast, three of the eight patients in groups II or III had a history of sustained VT.

\section{Discussion}

Propagation in patients with IDC. In seven (47\%) of the patients with IDC (group I) the pattern of activation and the 

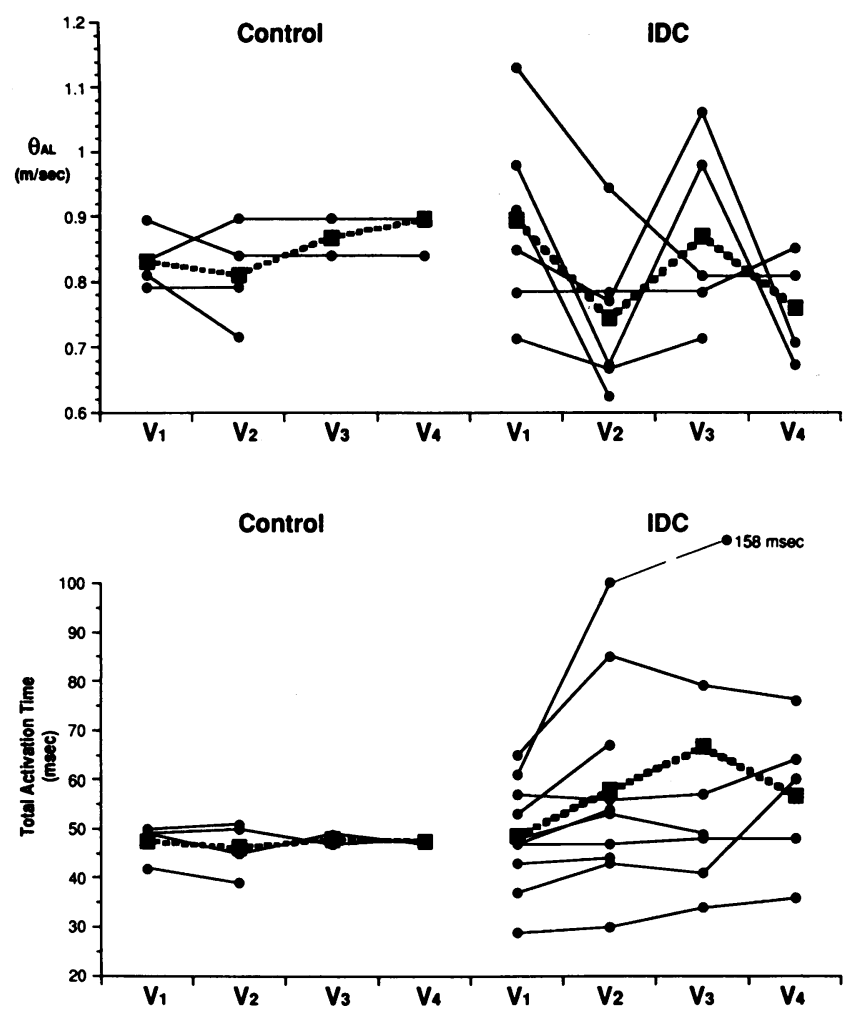

Figure 11. Effects of premature stimulation. Top panel: Longitudinal conduction velocity determined by method $\mathrm{A}\left(\theta_{\mathrm{AL}}\right)$ is plotted for the last beat in the eight beat train of stimuli $\left(V_{1}\right)$ and for the first $\left(V_{2}\right)$, second $\left(V_{3}\right)$, and third $\left(V_{4}\right)$ premature beats in control subjects (Control) and in patients with idiopathic dilated cardiomyopathy $(I D C)$. The results for individual patients are plotted as small filled circles $(\bullet)$. Mean values are indicated by filled squares $(\bullet)$ connected by thick dashed lines. Not all patients received three extrastimuli, so the mean values were obtained from different numbers of patients. The mean cycle length of the train $\left(V_{1}-V_{1}\right)$ was $475 \mathrm{msec}$ in controls and $440 \mathrm{msec}$ in patients with IDC. The mean values of $V_{2}, V_{3}$, and $V_{4}$ were 253,235 , and $230 \mathrm{msec}$, respectively, in control subjects, and 260, 236, and $220 \mathrm{msec}$, respectively, in patients with IDC. Bottom panel: TAT is plotted for each premature beat in each patient. The format is the same as in the top panel. The TAT of $V_{3}$ for one patient is beyond the scale of the plot and is indicated by a broken line and the TAT at that point given is provided.

longitudinal and transverse propagation velocities could not be distinguished from those obtained in patients without evidence of myocardial dysfunction. This does not prove, of course, that factors such as sodium conductance, membrane resistance, capacitance, and others discussed in connection with the mathematical model of propagation were not severely affected in this group. It is possible that a change in a factor which reduced $\theta$ was accompanied by alterations which increased $\theta$. However, it seems unlikely that the "compensating" factors would have corrected $\theta$ so precisely. It should be emphasized that the electrical recordings were made from a small region of myocardium so it is quite possible that abnormal propagation was present elsewhere in the hearts of this group of patients. Nevertheless, it seems improbable that contractility would be normal in the particular patch of left ventricular myocardium we chose to record. These findings suggest, therefore, that the functional and structural changes associated with severe contractile dys- function in patients with IDC are not sufficient to alter myocardial propagation in some patients with this disorder.

The four patients in group II had moderate disturbances in propagation which included disturbed transverse propagation associated with multiphasic electrograms and preserved longitudinal propagation. The observed activation sequences were not consistent with patterns $\mathrm{A}, \mathrm{B}, \mathrm{C}$, or D described in the methods section, and have not been reproduced by interventions which alter resting membrane potential $(39,44,50)$, sodium conductance (37-42), intracellular calcium (28), or uniform changes in gap junction resistance $(47,48)$. Instead, the findings indicate nonuniform anisotropic properties as defined by Spach et al. $(35,49)$, who put forth the explanation that such behavior results from reduced side-to-side electrical connections between muscle bundles. Although this must involve abnormal gap junction function, it differs from the changes resulting in pattern $D$ in that only gap junctions responsible for side-to-side propagation are assumed to be affected. The multiphasic nature of the electrograms during transverse propagation are explained by "bursts" of electrical activity arising from synchronous firing of adjacent well coupled myocytes within individual bundles, while intervals between bursts result from the additional time required to cross or circumvent the insulating boundaries between muscle groups. End-to-end connections between myocytes are presumed to be intact so that during longitudinal propagation adjacent bundles (even if poorly coupled side-to-side) are activated nearly simultaneously producing smooth extracellular waveforms $(35,49)$.

The spread of activation was severely disturbed in patients in group III. Marked fractionation of the electrograms and impaired transverse propagation was noted in this group, but unlike group II, longitudinal propagation was also abnormal. Longitudinally oriented insulating boundaries account for the multiphasic signals and reduced transverse conduction, but do not explain the changes in longitudinal activation. Although we cannot exclude abnormal membrane or cellular electrophysiologic properties, a more reasonable explanation might be a progression of the processes that caused the changes in group II. Large amounts of interstitial collagen, replacement fibrosis, and microscopic scars could have created obstacles to propagation. In addition, loss of the normal parallel arrangement probably contributed to the loss of a single discernable direction of longitudinal propagation in the activation maps (51).

Implications of abnormal propagation. An association between abnormal propagation and fibrosis has been described in atrial tissue $(26,64,65)$ and in animal models of myocardial infarction $(50,51)$, but not in patients or animal with cardiomyopathy. Nevertheless, the spectrum of histologic changes we observed is consistent with numerous previous studies of patients with IDC $(20,21,23)$, so there is reason to suspect that nonuniform anisotropic electrical properties are prevalent in this disorder.

Although disturbed propagation may contribute to the development of malignant ventricular tachyarrhythmias in patients with IDC, we know of no previous studies that have addressed this issue directly. There have been reports in various animal models of cardiomyopathies of associations between conduction abnormalities or fibrosis and ventricular arrhythmias or sudden death $(9,66-69)$ but none have examined myocardial propagation per se. A possible link between the observed changes in propagation and arrhythmogenesis was 


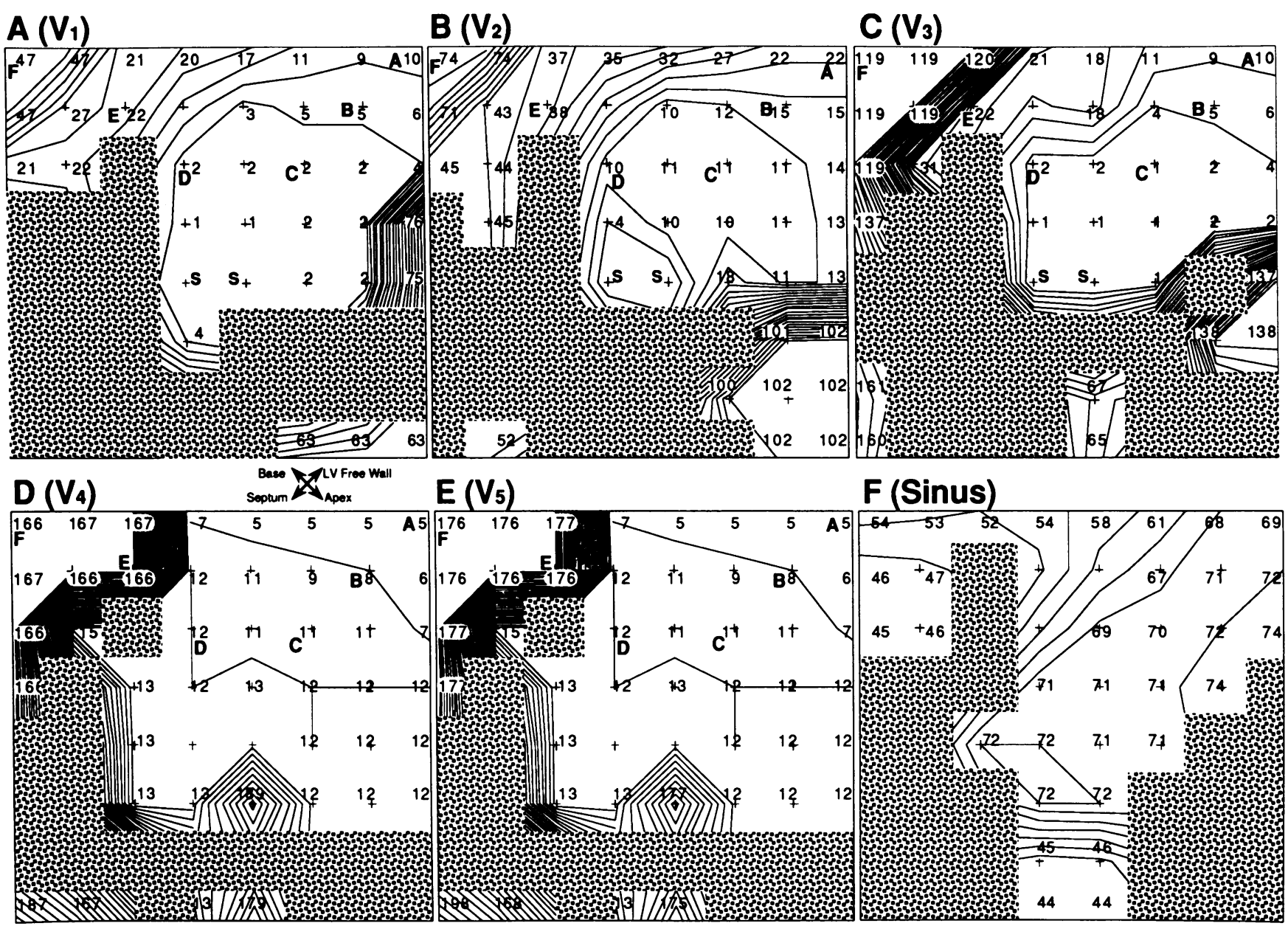

Figure 12. Activation patterns resulting from premature stimulation in a patient with IDC. Same format as in Fig. 7. The interval between isochrone contours is $4 \mathrm{~ms}$. Isochrones were drawn at 20 - $\mathrm{ms}$ intervals in areas where the gradient of activation times exceeded the capacity of the printer to generate distinct contours. The stimulation program consisted of a train of eight stimuli at a cycle length of $500 \mathrm{~ms}$ followed by three premature stimuli with coupling intervals of 260,230 , and $210 \mathrm{~ms}$. $(A)$ Activation map of the beat initiated by the last stimulus of the fixed-cycle length train $\left(V_{1}\right) .(B)$ Activation map of the beat initiated by the first premature stimulus $\left(V_{2}\right)$. $(C)$ Activation map of the beat initiated by the second premature stimulus $\left(V_{3}\right) .(D)$ Activation map of the first ventricular beat following the third premature stimulus $\left(V_{4}\right)$, but probably not initiated by it. (E) Activation map of the second ventricular beat following the third premature stimulus $\left(V_{5}\right)$. $(F)$ Activation map of a sinus beat. Activation times were referenced to the stimulus artifact for the stimulated beats $\left(V_{1}, V_{2}\right.$, and $\left.V_{3}\right)$ and to the onset of the QRS complex derived from the RMS of the recorded voltages for the nonstimulated beats $\left(V_{4}, V_{5}\right.$, and sinus). See text for discussion.

provided by Spach et al. (62) who demonstrated microreentry in dilated human atrial tissue characterized by nonuniform anisotropic properties but otherwise normal action potentials. Nonuniform anisotropy has also been reported in patients with VT (4), and is associated with reentry in the subacute and healed phases of myocardial infarction $(61,70)$. It is not known what degree of nonuniformity is required to alter vulnerability to arrhythmias (71). However, the speed of activation around a region of slow conduction or block is a determinant of reentry $(43,61)$. Therefore, the large gradients in activation times we observed may predispose to reentrant arrhythmias. In one case, premature stimulation associated with progressive conduction delays resulted in nonstimulated beats which appeared to arise close to the site of stimulation, a pattern consistent with, but not proof of, reentry (62). In any case, activation patterns and histologic findings in some patients with IDC are similar to those of the border zone of myocardial infarction which may indicate common elements between arrhythmias which occur after myocardial infarction and arrhythmias which occur in patients with $\operatorname{IDC}(51,61)$.

The presence of moderately disturbed propagation in two of the control patients is intriguing. Neither had evidence of systolic or diastolic dysfunction by history, physical examination or echocardiography, and neither had incessant supraventricular arrhythmias which could alter ventricular function. One of these patients had relatively frequent single PVCs, but this is sometimes seen among healthy subjects. While nonuniform anisotropy is known to occur in normal myocardium (49, 56 ), it has not been previously reported in this region of the heart and we have never observed it in this position of the canine left ventricle $(37,42)$. A relation between nonuniform properties and age has been noted (49). Although these two subjects ( 34 and $45 \mathrm{yr}$ ) were older than the average control subject, several patients with IDC and normal activation patterns were even older, including the oldest person in the study $(61 \mathrm{yr})$. Also, we found no correlation between age and the 


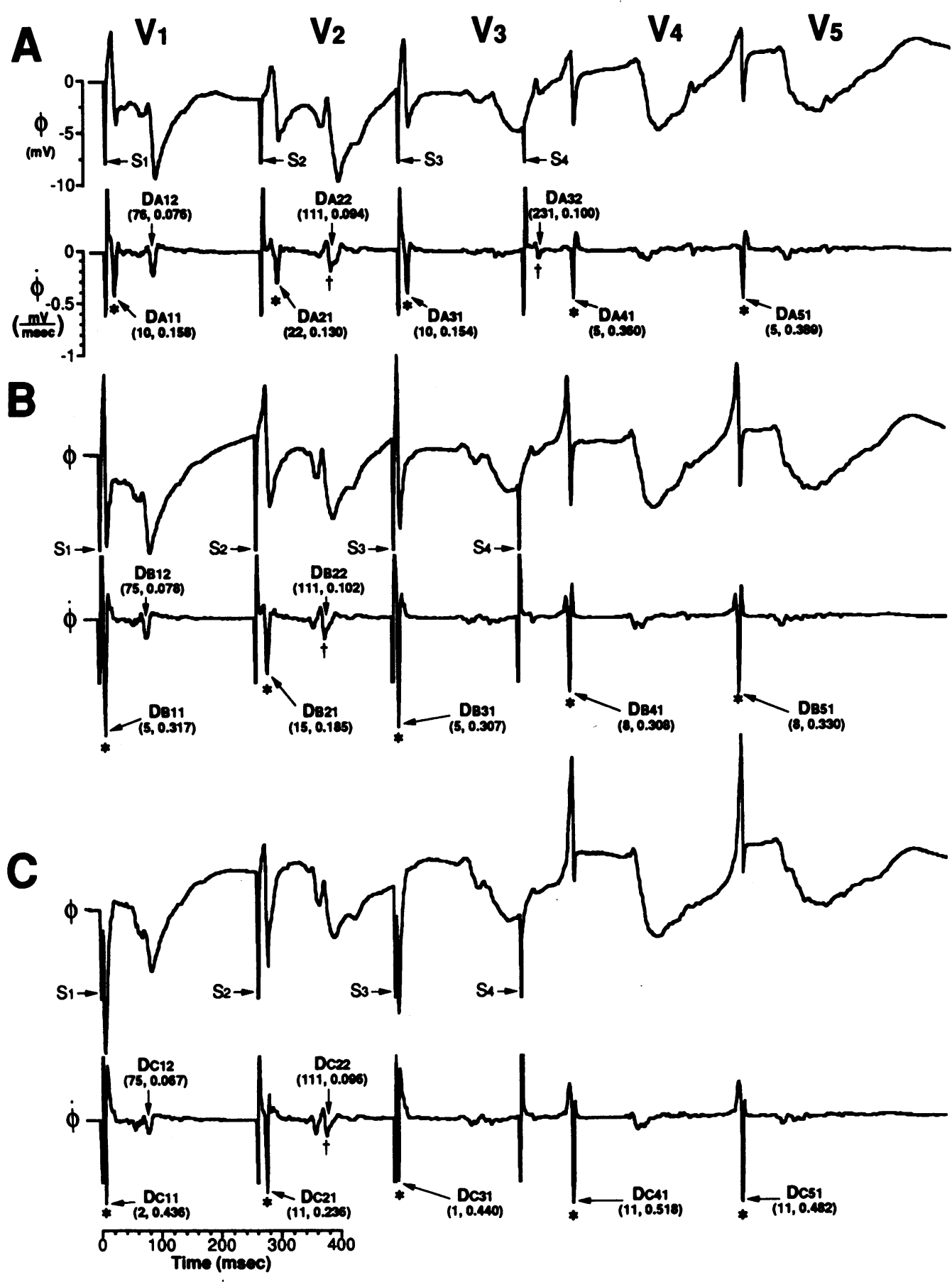

Figure 13. Unipolar electrograms $(\phi)$ and first derivative $(\dot{\phi})$ plots from recordings obtained at sites $A, B$, and $C$ (panels $A, B$, and $C$, respectively) in Fig. 12. The recordings are aligned in time and include the stimulus artifacts $\left(S_{1}, S_{2}, S_{3}\right.$, and $\left.S_{4}\right)$ and extracellular waveforms of the last beat of the fixedcycle length train $\left(V_{1}\right)$, the first and second stimulated beats $\left(V_{2}, V_{3}\right)$ and the two ventricular beats which followed the last stimulus $\left(V_{4}\right.$,

$\left.V_{5}\right) . S_{4}$ does not appear to initiate activation. Prominent deflections are labeled $D_{x y z}$ where $x$ is the electrode site, $y$ is the number of the associated beat $\left(1=V_{1}, 2=V_{2}\right.$, etc.), and $z$ is the number of the deflection, thus, $D_{\mathrm{A} 42}$ refers to the second deflection associated with $V_{4}$ recorded at site $A$. The first number in parentheses below each deflection label is the time of the deflection ( $\mathrm{msec}$ ); the second number is the value of $\psi$. Primary deflections, i.e., the deflection during a given beat which had the greatest value of $\psi$ and which met criteria for activation ( $\psi$ $\geq 0.093$ ) are indicated by an asterisk (*). Secondary deflections, i.e., deflections with smaller values of $\psi$ but which still met criteria for activation are indicated by a dagger $(\dagger)$. For $V_{1}, V_{2}$, and $V_{3}$ the reference (time $=0 \mathrm{msec}$ ) was the stimulus artifact that immediately precedes the beat. For $V_{4}$ and $V_{5}$ the referencewas the onset of the QRS derived from the root-meansquare voltage curve. The intervals are $S_{1}-S_{2}=260 \mathrm{msec}$, $S_{2}-S_{3}=230 \mathrm{msec}, S_{3}-S_{4}=210 \mathrm{msec}, S_{3}-V_{4}$ RMS curve $=230 \mathrm{msec}, V_{4}-V_{5}$ RMS curve $=283 \mathrm{msec}$. The activation order for $V_{1}$ is $C\left(D_{\mathrm{C} 11}\right) \rightarrow$ $B\left(D_{\mathrm{B} 11}\right) \rightarrow A\left(D_{\mathrm{A} 11}\right)$; the same order applies to $V_{2}$, and $V_{3}$. In contrast, the activation order for $V_{4}$ is reversed, i.e., $A\left(D_{\mathrm{A} 41}\right) \rightarrow B\left(D_{\mathrm{B} 11}\right) \rightarrow$ $C\left(D_{\mathrm{C} 1}\right)$, and $V_{\mathrm{s}}$ has a similar pattern.

degree of nonuniform activation (NAI), and previous studies have noted no correlation between myocardial fibrosis and age in patients with IDC (18). Therefore, it is not clear if the disturbed activation patterns detected in the two control subjects should be considered normal variants or abnormalities due to some undetermined cardiac disorder. Nevertheless, this finding demonstrates that nonuniform anisotropic properties can occur in the absence of demonstrable cardiac dysfunction. It seems possible that this abnormality might underlie ventricular tachyarrhythmias in some patients with "no structural heart disease." Because collagen infiltration is associated with nonuniform anisotropic propagation, the finding of interstitial fibrosis in a large percentage of patients with serious ventricular arrhythmias and no other evidence of cardiac disease may be relevant (72).

Limitations. There were a number of potential sources for error in this investigation. The model of myocardial propagation is relatively simple and is unlikely to simulate all the possible changes that could simultaneously occur in myopathic states, and it is not likely to reliably predict the impact of 


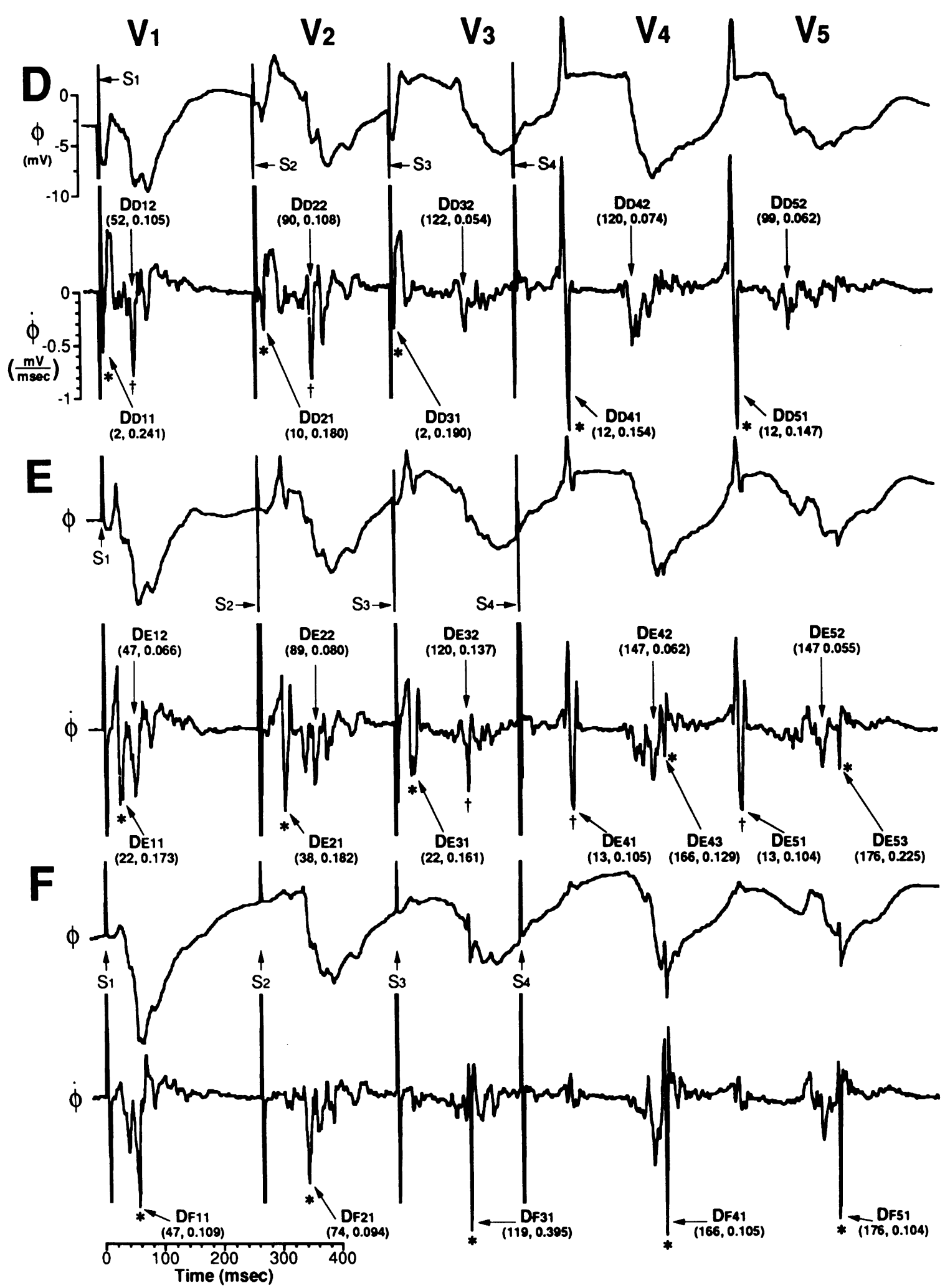

Figure 14. Unipolar electrograms $(\phi)$ and first derivative $(\dot{\phi})$ plots from recordings obtained at sites $D, E$, and $F$ (panels $D, E$, and $F$, respectively) in Fig. 12. Same format as Fig. 13. (D) Activation occurs early in each cycle at site $D$. The delay between primary and secondary deflections increases between $V_{1}$ and $V_{2}$. $(E)$ There are progressive temporal delays between primary and secondary deflections during beats $V_{3}, V_{4}$, and $V_{5}$ at this site. This site switches from being activated early in the cycle during $V_{1}$ and $V_{2}$ to being late during $V_{3}, V_{4}$, and $V_{5}$. This probably occurs because the myocyte bundles that contribute most to electrical activity at this site are subject to the progressive conduction delay. $(F)$ The progressive delay in activation is clearest at this site. 

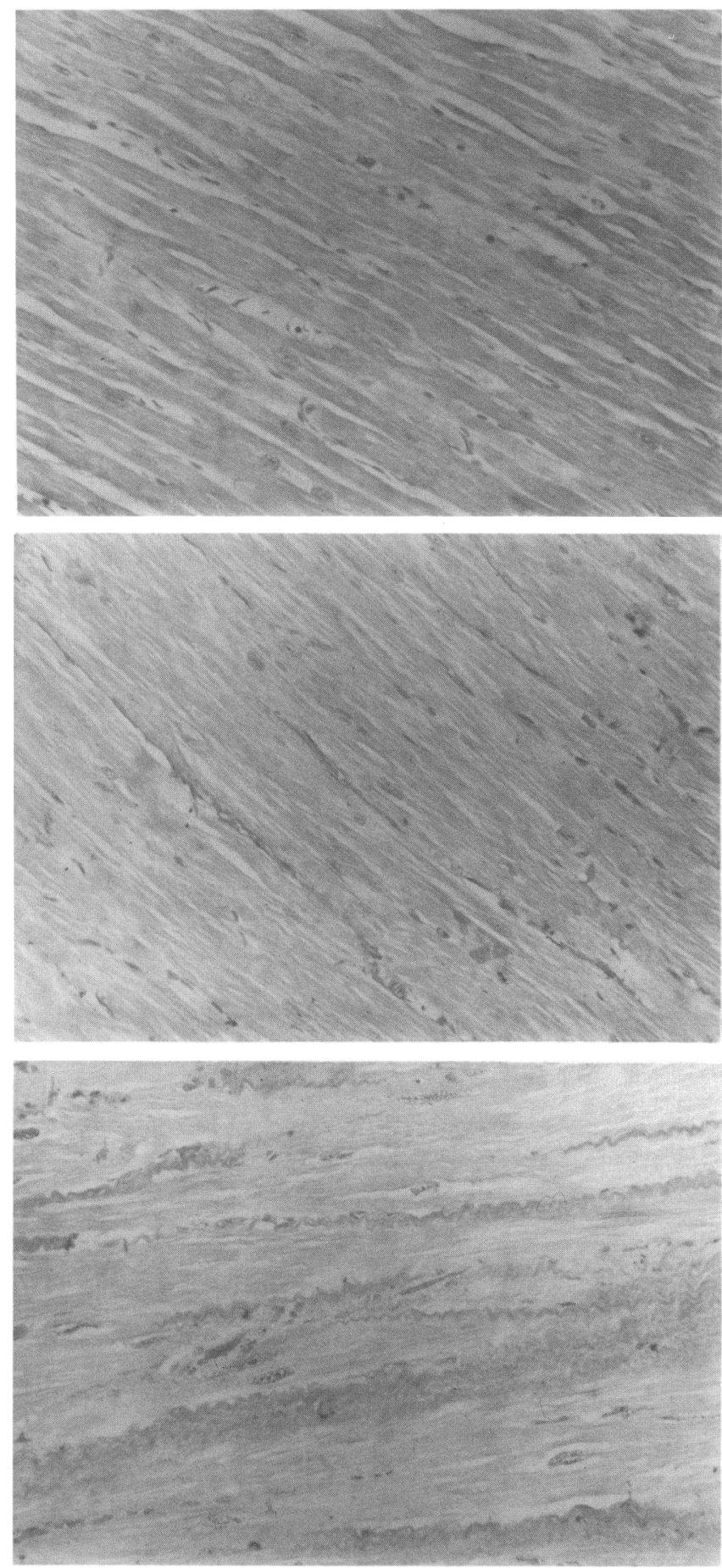

Figure 15. Photomicrographs of subepicardial myocardial tissue obtained from the region immediately beneath the plaque electrode array in patients with IDC. The sections were stained with Mallory's trichrome stain and magnified $\times 200$. Top Panel: Section from a 22yr-old man with IDC and minimal disturbance in activation sequence (group I). Middle panel: Section from a 35-yr-old man with IDC and moderately disturbed electrical activation (group II). Bottom panel: Section from 35-yr-old woman with IDC and severely disturbed activation sequence (group III).

changes in parameters which differ from cell to cell or region to region. The electrophysiologic data were collected in the anesthetized, open-chest state which cannot be considered "nor- mal" but neither the drugs used (narcotics and nondepolarizing paralytic agents) nor the physiologic changes are known to affect propagation in a significant manner. The finding of activation patterns in most of the control subjects and in a large proportion of IDC patients similar to those obtained in vivo $(37,42)$ and in vitro $(36,39,47,54,59)$ using a variety of anesthetic techniques suggests that the abnormal physiologic state did not have a major impact on the results. The methods of estimating effective conduction velocity assumed a linear path of conduction which could not be proved without knowledge of activation in three dimensions. However, deviation from a direct route is less likely to occur over the short distances used in this study and can usually be detected by careful examination of the superficial activation pattern. Moreover, the conduction velocities we obtained are comparable to those obtained in dogs $(37,42,54,59)$, pigs (36), rabbits (39), and sheep (47).

Time limitations restricted the number of interventions and measurements. The use of excised tissue would have allowed more precise control of the experimental milieu and more time to perform manipulations, but would not have been possible for the control subjects. In addition, the collection of electrophysiologic data in situ avoids several possible sources of artifacts. The loss of arterial perfusion, for instance, has been shown to increase extracellular resistance and reduce $\theta(73)$, while the presence of a tissue bath tends to reduce extracellular resistance which increases $\theta(31)$. Furthermore, it is widely recognized that investigations based on dissected tissue or isolated cells may favor healthier specimens (29). For example, Gwathmey et al. (74) acknowledged that the need to avoid tissue samples with dense necrosis or fibrosis could have accounted for the finding of normal isometric tension in human end-stage heart failure. Also, others have reported difficulty obtaining intracellular recordings in areas of dense fibrosis (51). In this regard, experiments performed in situ can complement data obtained in other conditions.

The electrophysiologic findings may not have been representative of changes present elsewhere in the heart. Although systolic dysfunction in patients with IDC is usually diffuse, the morphologic abnormalities may be heterogeneous (23). Fibrosis is reported to be greater in the left ventricular subendocardium and in the right ventricular segments than in the left ventricular epicardial region where our measurements were obtained (20). Therefore, if fibrosis is a marker for nonuniform anisotropic properties, one could expect even greater disturbances of conduction elsewhere in the heart and the finding of normal propagation in some of the patients does not exclude the presence of severe disturbances in other areas.

Clinical implications. The tendency for more frequent and complex ventricular arrhythmias in the patients with perturbed activation and increased fibrosis must be interpreted cautiously, because, as noted above, the patients with normal appearing propagation in the sampled region could have had severe abnormalities elsewhere. Furthermore, if conduction disturbances do play a role in arrhythmogenesis in patients with IDC, it is unlikely to be exclusive. Nonuniform anisotropic properties probably interact with an array of changes such as increased dispersion of refractoriness, altered calcium kinetics, and increased sympathetic activity to increase the vulnerability to malignant ventricular arrhythmias. For instance, ventricular premature beats triggered by depolarizations result- 
ing from abnormal calcium movements could precipitate reentry in tissue with nonuniform anisotropic properties. Nevertheless, if nonuniform anisotropic propagation and fibrosis are important elements in the development of arrhythmias in patients with IDC, then they may also be important in other disorders characterized by pathological collagen deposition such as hypertrophic cardiomyopathy, hypertensive cardiovascular disease, and other forms of cardiomyopathy. A relation between fibrous tissue and arrhythmias also may have therapeutic implications. Treatment with angiotensin-converting enzyme inhibitors and calcium antagonists has been shown to limit the development of fibrosis in animal models of cardiomyopathy $(9,75-77)$. Therefore, inhibition of collagen infiltration with failure to develop nonuniform propagation might be one mechanism by which these agents reduce mortality in animals with cardiomyopathy $(9,78)$ and humans with congestive heart failure $(79,80)$.

\section{Acknowledgments}

The authors thank Drs. Michael Bristow, John O'Connell, and Dale Renlund and other members of the Utah Cardiac Transplantation Program for their support and encouragement, Donna Runnels, R.N., and Susan Lyver, R.N., for coordinating the clinical aspects of the investigation, and Judy Wintch for secretarial assistance.

This work was supported by a grant-in-aid from the American Heart Association, and by a grant from the Dr. Ezekiel R. and Edna Wattis Dumke Foundation. Dr. Anderson was supported by a FIRST award HL-34198 from the National Institutes of Health.

\section{References}

1. Meinertz, T., T. Hofmann, W. Kasper, N. Treese, H. Bechtold, U. Stienen, T. Pop, E. V. Leitner, D. Andresen, and J. Meyer. 1984. Significance of ventricular arrhythmias in idiopathic dilated cardiomyopathy. Am. J. Cardiol. 53:902907.

2. Unverferth, D. V., R. D. Magorien, M. L. Moeschberger, P. B. Baker, J. K. Fetters, and C. V. Leier. 1984. Factors influencing the one-year mortality of dilated cardiomyopathy. Am. J. Cardiol. 54:147-152.

3. Chakko, C. S., and M. Gheorghiade. 1989. Ventricular arrhythmias in severe heart failure: incidence significance and effectiveness of antiarrhythmic therapy. Am. Heart J. 109:497-503.

4. de Bakker, A. M. T., R. Coronel, S. Tasseron, A. A. M. Wilde, T. Opthof M. J. Janse, F. J. L. van Capelle, A. E. Becker, and G. Jambroes. 1990. Ventricular tachycardia in the infarcted Langendorff-perfused human heart: role of the arrangement of surviving cardiac fibers. J. Am. Coll. Cardiol. 15:1594-1607.

5. Poll, D. S., F. E. Marchlinski, R. A. Falcone, M. E. Josephson, and M. B. Simson. 1985. Abnormal signal-averaged electrocardiograms in patients with nonischemic congestive cardiomyopathy: relationship to sustained ventricular tachyarrhythmias. Circulation. 72:1308-1313.

6. Middlekauff, H. R., W. G. Stevenson, M. A. Woo, D. K. Moser, and L. W. Stevenson. 1990. Comparison of frequency of late potentials in idiopathic dilated cardiomyopathy and ischemic cardiomyopathy with advanced congestive heart failure and their usefulness in predicting sudden death. Am. J. Cardiol. 66:11131117.

7. Ohnishi, T., T. Inoue, and H. Fukuzaki. 1990. Value of the signal-averaged electrocardiogram as a predictor of sudden death in myocardial infarction and dilated cardiomyopathy. Jpn. Circ. J. 54:127-136.

8. Gonska, B. D., K. P. Bethge, H. R. Gigulla, and H. Kreuzer. 1988. Occurrence and clinical significance of endocardial late potentials and fractionations in idiopathic dilated cardiomyopathy. Br. Heart J. 59:39-46.

9. Fein, F. S., J. M. Capasso, R. S. Aronson, S. Cho, C. Nordin, B. MillerGreen, E. H. Sonnenblick, and S. M. Factor. 1984. Combined renovascular hypertension and diabetes in rats: a new preparation of congestive cardiomyopathy. Circulation. 70:318-330.

10. Rossner, K. L., and H. G. Sachs. 1978. Electrophysiological study of Syrian hamster hereditary cardiomyopathy. Cardiovasc. Res. 12:436-443.

11. Einzig, S., B. L. Detloff, B. K. Borgwardt, N. A. Staley, G. R. Noren, and D. G. Benditt. 1981. Cellular electrophysiological changes in "round heart dis- ease" of turkeys: a potential basis for dysrhythmia in myopathic ventricles. Cardiovasc. Res. 15:643-651.

12. Sen, L., M. O'Neill, J. D. Marsh, and T. W. Smith. 1990. Inotropic and calcium kinetic effects of calcium channel agonist and antagonist in isolated cardiac myocytes from cardiomyopathic hamsters. Circ. Res. 67:599-608.

13. Keung, E. C., L. Toll, M. Ellis, and R. A. Jensen. 1991. L-type cardiac calcium channels in doxorubicin cardiomyopathy in rats morphological, biochemical, and functional correlations. J. Clin. Invest. 87:2108-2113.

14. Ferrans, V. J., R. A. Massumi, G. I. Shugoll, N. Ali, and W. C. Roberts. 1973. Ultrastructural studies of myocardial biopsies in 45 patients with obstructive or congestive cardiomyopathy. In Recent Advances in Studies on Cardiac Structure and Metabolism. Volume II: Cardiomyopathies. E. Bajusz and G. Rona, editors. University Park Press, Baltimore, MD. 231-272.

15. Kunkel, B., H. Lapp, G. Kober, and M. Kaltenbach. 1978. Correlations between clinical and morphologic findings and natural history in congestive cardiomyopathy. In Cardiomyopathy and Myocardial Biopsy. M. Kaltenbach, F. Loogen, and E. G. J. Olsen, editors. Springer-Verlag, Berlin. 271-283.

16. Anderson, K. R., and M. G. S. J. Sutton. 1979. Histopathological types of cardiac fibrosis in myocardial disease. J. Pathol. 128:79-85.

17. Baandrup, U., R. A. Florio, M. Rehahn, P. J. Richardson, and E. G. J. Olsen. 1981. Critical analysis of endomyocardial biopsies from patients suspected of having cardiomyopathy. II. Comparison of histology and clinical/haemodynamic information. Br. Heart J. 45:487-493.

18. Unverferth, D. V., J. K. Feeters, B. J. Unverferth, C. V. Leier, R. D. Magorien, A. R. Arn, and P. B. Baker. 1983. Human myocardial histologic characteristics in congestive heart failure. Circulation. 68:1194-1200.

19. Rose, A. G., and W. Beck. 1985. Dilated (congestive) cardiomyopathy: a syndrome of severe cardiac dysfunction with remarkably few morphological features of myocardial damage. Histopathology. 9:367-379.

20. Unverferth, D. V., P. B. Baker, S. E. Swift, R. Chaffee, J. K. Fetters, B. F Uretsky, M. E. Thompson, and C. V. Leier. 1986. Extent of myocardial fibrosis and cellular hypertrophy in dilated cardiomyopathy. Am. J. Cardiol. 57:816-820.

21. Roberts, W. C., R. J. Siegel, and B. M. McManus. 1987. Idiopathic dilated cardiomyopathy: analysis of 152 necropsy patients. Am. J. Cardiol. 60:1340 1355.

22. Schaper, J., R. Froede, S. Hein, A. Buck, H. Hashizume, B. Speiser, A Friedl, and N. Bleese. 1991. Impairment of the myocardial ultrastructure and changes of the cytoskeleton in dilated cardiomyopathy. Circulation. 83:504-514.

23. Maehashi, N., Y. Yokota, A. Takarada, S. Usuki, S. Maeda, H. Yoshida, T. Sugiyama, and H. Fukuzaki. 1991. The role of myocarditis and myocardial fibrosis in dilated cardiomyopathy: analysis of 28 necropsy cases. Jpn. Heart J. 32:1-15.

24. Baandrup, U., R. A. Florio, F. Rotes, and E. G. J. Olsen. 1981. Electron microscopic investigation of endomyocardial biopsy samples in hypertrophy and cardiomyopathy. Circulation. 63:1289-1297.

25. Keener, J. P. 1991. The effects of discrete gap junction coupling on propagation in myocardium. J. Theor. Biol. 148:49-82.

26. Dolber, P. C., and M. S. Spach. 1989. Structure of canine Bachmann's bundle related to propagation of excitation. Am. J. Phvsiol. 257:H1446-H1457.

27. Fozzard, H. A., and M. F. Arnsdorf. 1986. Cardiac electrophysiology. In The Heart and Cardiovascular System: Scientific Foundations. H. A. Fozzard, E Haber, R. B. Jennings, A. M. Katz, and H. E. Morgan, editors. Raven Press, New York: $1-30$.

28. Spach, M. S., J. M. Kootsey, and J. D. Sloan. 1982. Active modulation of electrical coupling between cardiac cells of the dog. Circ. Res. 51:347-362.

29. Sommer, J. R., and Jennings, R. B. Ultrastructure of cardiac muscle. In The Heart and Cardiovascular System: Scientific Foundations. H. A. Fozzard, E. Haber, R. B. Jennings, A. M. Katz, and H. E. Morgan, editors. Raven Press, New York. 3-50.

30. Weidmann, S. 1970. Electrical constants of trabecular muscle from mammalian heart. J. Physiol. (Lond.). 210:1041-1054.

31. Roth, B. J. 1991. Action potential propagation in a thick strand of cardiac muscle. Circ. Res. 68:162-173.

32. Fozzard, H. A. 1966. Membrane capacity of the cardiac Purkinje fibers. $J$. Physiol. (Lond.). 182:255-267.

33. Woodbury, J. W., and W. E. Crill. 1961. On the problem of impulse conduction in the atrium. In Nervous Inhibition. E. Florey, editor. Pergamon Press, New York. 124-135.

34. Chapman, R. A., and C. H. Fry. 1978. An analysis of the cable properties of frog ventricular myocardium. J. Physiol. (Lond.). 283:263-282.

35. Spach, M. S., W. T. Miller III, E. Miller-Jones, R. B. Warren, and R. C Barr. 1979. Extracellular potentials related to intracellular action potentials during impulse conduction in anisotropic canine cardiac muscle. Circ. Res. 45:188 204.

36. Kléber, A. G., M. J. Janse, F. J. G. Wilms-Schopmann, A. A. M. Wilde, and $\mathbf{R}$. Coronel. 1986. Changes in conduction velocity during acute ischemia in ventricular myocardium of the isolated porcine heart. Circulation. 73:189-198.

37. Anderson, K. P., R. Walker, T. Dustman, R. L. Lux, P. R. Ershler, R. E. Kates, and P. M. Urie. 1989. Rate-related electrophysiologic effects of long-term 
amiodarone administration on canine ventricular myocardium in vivo. Circula tion. 79:948-958.

38. Spach, M. S., P. C. Dolber, J. F. Heidlage, J. M. Kootsey, and E. A Johnson. 1987. Propagating depolarization in anisotropic human and canine cardiac muscle: apparent directional differences in membrane capacitance. Circ. Res. 60:206-219.

39. Brugada, J., L. Mont, L. Boersma, C. Kirchhof, and M. A. Allessie. 1991. Differential effects of heptanol, potassium, and tetrodotoxin on reentrant ventricular tachycardia around a fixed obstacle in anisotropic myocardium. Circulation. 84:1307-1318.

40. Kadish, A. H., J. F. Spear, J. H. Levine, and E. N. Moore. 1986. The effects of procainamide on conduction in anisotropic canine ventricular myocardium. Circulation. 74:616-625.

41. Bajaj, A. K., H. A. Kopelman, J. P. Wikswo, F. Cassidy, R. L. Woosley, and D. M. Roden. 1987. Frequency- and orientation-dependent effects of mexiletine and quinidine on conduction in the intact dog heart. Circulation. 75:10651073.

42. Anderson, K. P., R. Walker, R. L. Lux, P. R. Ershler, R. Menlove, M. R. Williams, R. Krall, and D. Moddrelle. 1990. Conduction velocity depression and drug-induced ventricular tachyarrhythmias: effects of lidocaine in the intact canine heart. Circulation. 81:1024-1038.

43. Wit, A. L., and S. M. Dillon. 1990. Anisotropic Reentry. In Cardiac Electrophysiology: from Cell to Bedside. D. P. Zipes, and J. Jalife, editors. W. B. Saunders Co., Philadelphia. 353-364.

44. Tsuboi, N., I. Kodama, J. Toyama, and K. Yamada. 1985. Anisotropic conduction properties of canine ventricular muscles: influence of high extracellular K+ concentration and stimulation frequency. Jap. Circ. J. 49:487-498.

45. Lo, Y. S. A., M. Billingham, R. A. Rowan, H. C. Lee, L. B. Liem, and C. D. Swerdlow. 1989. Histopathologic and electrophysiologic correlations in idiopathic dilated cardiomyopathy and sustained ventricular tachyarrhythmias. Am. J. Cardiol. 64:1063-1066.

46. Sommer, J. R., E. Bossen, H. Dalen, P. Dolber, T. High, P. Jewett, E. A. Johnson, J. Junker, S. Leonard, R. Nassar, et al. 1991. To excite a heart: a bird's view. Acta Physiol. Scand. 142:5-21.

47. Delmar, M., D. C. Michaels, T. Johnson, and J. Jalife. 1987. Effects of increasing intercellular resistance on transverse and longitudinal propagation in sheep epicardial muscle. Circ. Res. 60:780-785.

48. Balke, C. W., M. D. Lesh, J. F. R. Spear, A. Kadish, J. H. Levine, and E. N. Moore. 1988. Effects of cellular uncoupling on conduction in anisotropic canine ventricular myocardium. Circ. Res. 63:879-892.

49. Spach, M. S., and P. C. Dolber. 1986. Relating extracellular potentials and their derivatives to anisotropic propagation at a microscopic level in human cardiac muscle: evidence for electrical uncoupling of side-to-side fiber connections with increasing age. Circ. Res. 58:356-371.

50. Gardner, P., P. C. Ursell, J. J. Fenoglio, Jr., and A. L. Wit. 1985. Electrophysiological and anatomical basis for fractionated electrograms recorded from healed myocardial infarcts. Circulation. 72:596-611.

51. Ursell, P. C., P. I. Gardner, A. Albala, J. J. Fenoglio, Jr., and A. L. Wit. 1985. Structural and electrophysiological changes in the epicardial border zone of canine myocardial infarcts during infarct healing. Circ. Res. 56:436-451.

52. Ershler, P. R., R. L. Lux, and B. W. Steadman. 1986. A 128 lead online intraoperative mapping system. Invited paper for IEEE Engineering in Medicine and Biology Society's 8th Annual Conference, Ft. Worth, TX, 7-10 November 1986. 1289-1291.

53. Anderson, K. P., R. Walker, P. R. Ershler, M. Fuller, T. Dustman, R. Menlove, S. V. Karwandee, and R. L. Lux. 1991. Determination of local myocardial electrical activation for activation sequence mapping: a statistical approach. Circ. Res. 69:898-917.

54. Spach, M. S., and P. C. Dolber. 1989. Anisotropic discontinuous propagation: directional differences in distribution of gap junctions and in effective membrane capacitance of cardiac muscle. In Cell Interactions and Gap Junctions. N. Sperelakis and W. C. Cole, editors. CRC Press, Boca Raton, FL. 95-121.

55. Armour, J. A., and W. C. Randall. 1970. Structural basis for cardiac function. Am. J. Physiol. 218:1517-1523.

56. Burgess, M. J., B. M. Steinhaus, K. W. Spitzer, and P. R. Ershler. 1988 Nonuniform epicardial activation and repolarization properties of in vivo canine pulmonary conus. Circ. Res. 62:233-246.

57. Crawford, F. S., Jr. 1965. Waves: Berkeley Physics Course. Volume 3. McGraw-Hill Book Co., New York. 332-335.

58. Spear, J. F., C. W. Balke, M. D. Lesh, A. H. Kadish, J. H. Levine, and E. N. Moore. 1990. Effect of cellular uncoupling by heptanol on conduction in infarcted myocardium. Circ. Res. 66:202-217.

59. Wikswo, J. P., Jr., T. A. Wisialowski, W. A. Altemeier, J. R. Balser, H. A.
Kopelman, and D. M. Roden. 1991. Virtual cathode effects during stimulation of cardiac muscle: two-dimensional in vivo experiments. Circ. Res. 68:513-530.

60. Kraemer, H. 1988. Assessment of $2 \times 2$ associations: generalization of signal-detection methodology. Am. Stat. 42:42-49.

61. Dillon, S. M., M. A. Allessie, P. C. Ursell, and A. L. Wit. 1988. Influences of anisotropic tissue structure on reentrant circuits in the epicardial border zone of subacute canine infarcts. Circ. Res. 63:182-206.

62. Spach, M. S., P. C. Dolber, and J. F. Heidlage. 1988. Influence of the passive anisotropic properties on directional differences in propagation following modification of the sodium conductance in human atrial muscle: a model of reentry based on anisotropic discontinuous propagation. Circ. Res. 62:811-832.

63. Burgess, M. J., R. L. Lux, P. R. Ershler, and R. Menlove. 1990. Determination of transmural location of onset of activation from cardiac surface electrograms. Circulation. 82:1335-1342.

64. Spach, M. S., and P. C. Dolber. 1985. The relation between discontinuous propagation in anisotropic cardiac muscle and the "vulnerable period" of reen try. In Cardiac Electrophysiology and Arrhythmias. D. P. Zipes and J. Jalife, editors. Grune \& Stratton, Orlando, FL. 241-252.

65. Spach, M. S., W. T. Miller III, P. C. Dolber, J. M. Kootsey, J. R. Sommer, and C. E. Mosher, Jr. 1982. The functional role of structural complexities in the propagation of depolarization in the atrium of the dog: cardiac conduction disturbances due to discontinuities of effective axial resistivity. Circ. Res. 50:175191.

66. Bakth, S., J. Arena, W. Lee, R. Torres, B. Haider, B. C. Patel, M. M Lyons, and T. J. Regan. 1986. Arrhythmia susceptibility and myocardial composition in diabetes. J. Clin. Invest. 77:382-395.

67. Hano, O., T. Mitsuoka, Y. Matsumoto, R. Ahmed, M. Hirata, T. Hirata, M. Mori, K. Yano, and K. Hashiba. 1991. Arrhythmogenic properties of the ventricular myocardium in cardiomyopathic Syrian hamster, BIO 14.6 strain. Cardiovasc. Res. 25:49-57.

68. Weber, K. T., R. Pick, M. A. Silver, G. W. Moe, J. S. Janicki, I. H. Zucker, and P. W. Armstrong. 1990. Fibrillar collagen and remodeling of dilated canine left ventricle. Circulation. 82:1387-1401.

69. Li, H. G., D. L. Jones, R. Yee, and G. J. Klein. 1992. Electrophysiologic substrate associated with pacing-induced heart failure in dogs: potential value of programmed stimulation in predicting sudden death. J. Am. Coll. Cardiol. 19:444-449.

70. Zuanetti, G., R. H. Hoyt, and P. B. Corr. 1990. $\beta$-Adrenergic-mediated influences on microscopic conduction in epicardial regions overlying infarcted myocardium. Circ. Res. 67:284-302.

71. Lesh, M. D., J. F. Spear, and E. N. Moore. 1990. Myocardial anisotropy: Basic electrophysiology and role in cardiac arrhythmias. In Cardiac Electrophysiology: from Cell to Bedside. D. P. Zipes and J. Jalife, editors. W. B. Saunders Co. Philadelphia, 364-376.

72. Sugrue, D. D., D. R. Holmes, B. J. Gersh, W. D. Edwards, C. J. McLaran, D. L. Wood, J. M. Osborn, and S. C. Hammill. 1984. Cardiac histologic findings in patients with life-threatening ventricular arrhythmias of unknown origin. $J$. Am. Coll. Cardiol. 4:952-957.

73. Kléber, A. G., and C. B. Riegger. 1986. Electrical constants of arterially perfused rabbit papillary muscle. J. Physiol. (Lond.). 385:307-324.

74. Gwathmey, J. K., L. Copelas, R. MacKinnon, F. J. Schoen, M. D. Feldman, W. Grossman, and J. P. Morgan. 1987. Abnormal intracellular calcium handling in myocardium from patients with end-stage heart failure. Circ. Res. 61:70-76.

75. Tan, L.-B., J. E. Jalil, R. Pick, J. S. Janicki, and K. T. Weber. 1991. Cardiac myocyte necrosis induced by angiotensin II. Circ. Res. 69:1185-1195.

76. Kobayashi, A., T. Yamashita, M. Kaneko, T. Nishiyama, H. Hayashi, and N. Yamazaki. 1987. Effects of verapamil on experimental cardiomyopathy in the Bio 14.6 Syrian hamster. J. Am. Coll. Cardiol. 10:1128-1134.

77. Weber, K. T., and C. G. Brilla. 1991. Pathological hypertrophy and cardiac interstitium: fibrosis and renin-angiotensin-aldosterone system. Circulation. 83:1849-1865.

78. Haleen, S. J., R. E. Weishaar, R. W. Overhiser, R. F. Bousley, J. A. Keiser, S. R. Rapundalo, and D. G. Taylor. 1991. Effects of quinapril, a new angiotensin converting enzyme inhibitor, on left ventricular failure and survival in the cardiomyopathic hamster: hemodynamic, morphological, and biochemical correlates. Circ. Res. 68:1302-1312.

79. Cohn, J. N., G. Johnson, S. Ziesche, F. Cobb, G. Francis, F. Tristani, R Smith, W. B. Dunkman, H. Loeb, M. Wong, et al. 1991. A comparison of enalapril with hydralazine-isosorbide dinitrate in the treatment of chronic congestive heart failure. N. Engl. J. Med. 325:303-310.

80. Lee, W. H., and M. Packer. 1986. Prognostic importance of serum sodium concentration and its modification by converting-enzyme inhibition in patients with severe chronic heart failure. Circulation. 73:257-267. 